\title{
Tunneling splittings of vibrationally excited states using general instanton paths
}

Cite as: J. Chem. Phys. 153, 134106 (2020); https://doi.org/10.1063/5.0024210

Submitted: 05 August 2020 . Accepted: 08 September 2020 . Published Online: 01 October 2020

Mihael Eraković (D) and Marko T. Cvitaš (iD)
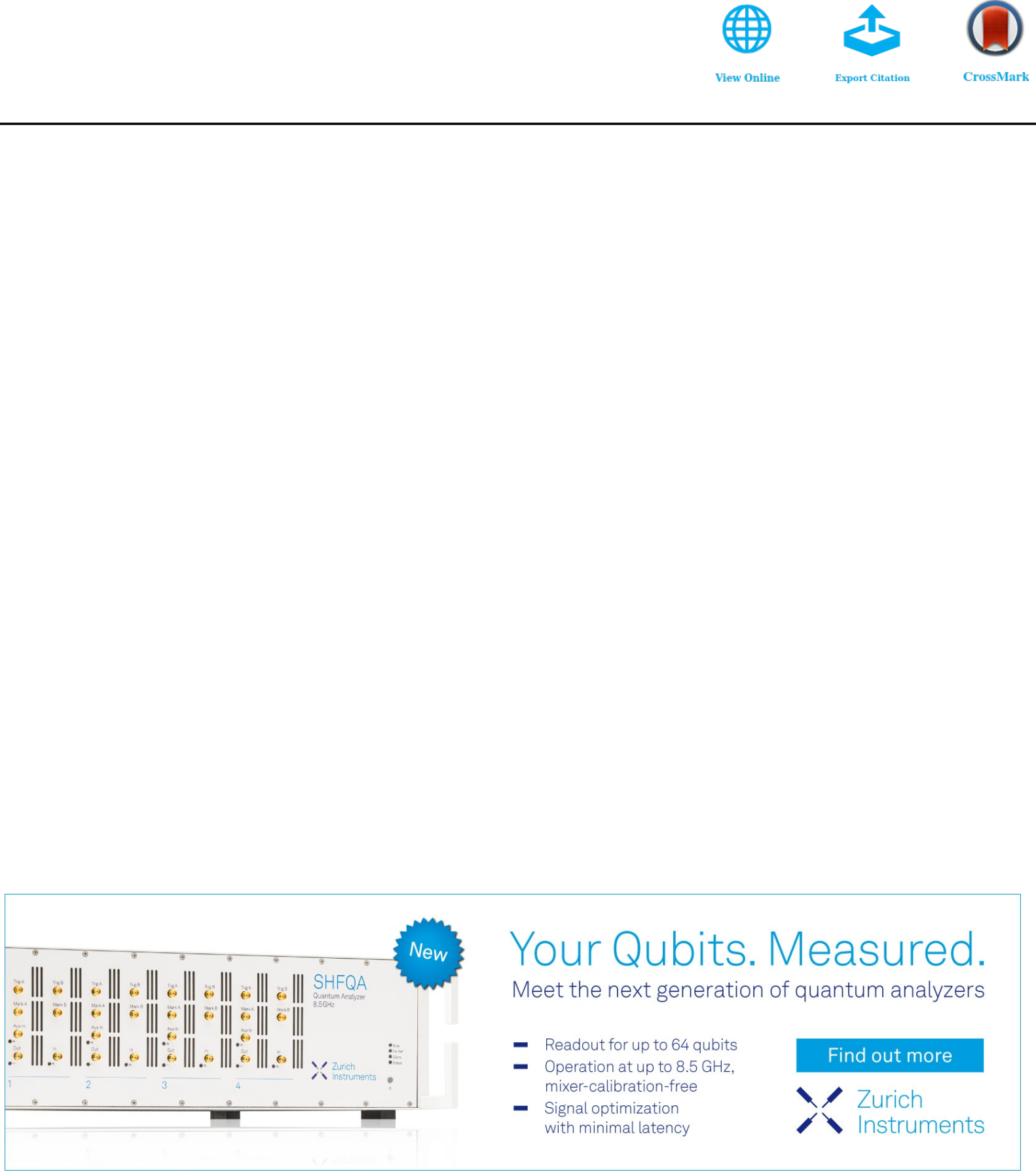


\title{
Tunneling splittings of vibrationally excited states using general instanton paths
}

\author{
Cite as: J. Chem. Phys. 153, 134106 (2020); doi: 10.1063/5.0024210 \\ Submitted: 5 August 2020 - Accepted: 8 September 2020 • \\ Published Online: 1 October 2020
}

\author{
Mihael Eraković (iD) and Marko T. Cvitaša
}

\section{AFFILIATIONS}

Department of Physical Chemistry, Ruđer Bošković Institute, Bijenička Cesta 54, 10000 Zagreb, Croatia

\author{
a) Author to whom correspondence should be addressed: mcvitas@irb.hr
}

\begin{abstract}
A multidimensional semiclassical method for calculating tunneling splittings in vibrationally excited states of molecules using Cartesian coordinates is developed. It is an extension of the theory by Mil'nikov and Nakamura [J. Chem. Phys. 122, 124311 (2005)] to asymmetric paths that are necessary for calculating tunneling splitting patterns in multi-well systems, such as water clusters. Additionally, new terms are introduced in the description of the semiclassical wavefunction that drastically improves the splitting estimates for certain systems. The method is based on the instanton theory and builds the semiclassical wavefunction of the vibrationally excited states from the ground-state instanton wavefunction along the minimum action path and its harmonic neighborhood. The splittings of excited states are thus obtained at a negligible added numerical effort. The cost is concentrated, as for the ground-state splittings, in the instanton path optimization and the hessian evaluation along the path. The method can thus be applied without modification to many mid-sized molecules in full dimensionality and in combination with on-the-fly evaluation of electronic potentials. The tests were performed on several model potentials and on the water dimer.
\end{abstract}

Published under license by AIP Publishing. https://doi.org/10.1063/5.0024210

\section{INTRODUCTION}

Tunneling splittings of molecular energy levels are spectroscopic signatures of rearrangements that take place between degenerate symmetric wells via tunneling motion. ${ }^{1-3}$ These splittings can be detected in high-precision spectroscopic measurements ${ }^{4,5}$ and carry information about the molecular structure and dynamics along the accessible tunneling paths. ${ }^{1,6}$ Dynamical theories, in combination with potential energy surfaces (PESs) or first principles electronic structure calculations, aim to reach an agreement with the measurements and provide a physical interpretation.

Computational studies of tunneling splittings concentrated initially on the symmetric tunneling systems. Proton transfer in malonaldehyde, ${ }^{8}$ collective migration of hydrogen atoms in ammonia, ${ }^{9}$ or concerted monomer motion in the HF dimer ${ }^{10}$ are some examples of extensively studied systems. More recently, the splitting patterns in water clusters ${ }^{7}$ have also come into focus, motivated by the development of a universal water model that is capable of predicting properties of liquid water from first principles. ${ }^{11-13}$ Water clusters are multi-well systems and exhibit multiple tunneling pathways. These tunneling paths are often asymmetric, whereby tunneling atoms take on different roles in the minima they connect. ${ }^{3}$

The splittings vary over many orders of magnitude even in a single system. In water dimer, for instance, they vary over three orders of magnitude ${ }^{14}$ depending on which of the five tunneling pathways is taken, all of which reflect on the appearance of the splitting pattern in the spectrum. Likewise, the experiments on water trimer $^{15}$ and pentamer ${ }^{16}$ show that the splittings of vibrationally excited states differ by up to three orders of magnitude in comparison to the ground-state splittings, depending on which normal mode is excited. The interplay of different rearrangement pathways can lead to an increase in the width of a vibrational manifold and a reduction in another ${ }^{14,17}$ as contributions from different pathways enter the splitting pattern with the same or opposite signs, respectively. Qualitatively different tunneling splitting patterns in the water hexamer spectrum distinguish the prism and cage structures $^{18,19}$ of almost equal energy. The contributions of different tunneling pathways can be disentangled, by computation, to reveal the experimental evidence of unexpected mechanisms, such as the simultaneous double hydrogen-bond breaking ${ }^{20}$ in the water hexamer prism. The investigations of tunneling splitting 
patterns thus provide a sensitive test of both the dynamical theories and the potentials at geometries along which the hydrogen bonds rearrange.

Tunneling splittings can be determined by solving the Schrödinger equation. Variational methods have been used to determine the tunneling splittings, e.g., in HF dimer, ${ }^{10}$ ammonia, ${ }^{9,21,22}$ vinyl radical, ${ }^{23}$ malonaldehyde, ${ }^{24}$ and water dimer, ${ }^{25,26}$ using timeindependent methods, and, e.g., in malonaldehyde, ${ }^{8,27-29}$ using timedependent methods. Both, ground- and excited-state splittings are obtained in this way; however, the cost of these methods scales prohibitively with the basis set size, and a different approach is needed for larger systems. Diffusion Monte Carlo in combination with the projection operator techniques has been used to calculate tunneling splittings in water trimer ${ }^{30}$ and malonaldehyde. ${ }^{31,32}$ The recently developed path-integral molecular dynamics method has been used to obtain the splittings in water trimer and hexamer ${ }^{33}$ in full dimensionality. However, the tunneling splittings of vibrationally excited states, which are the topic of our investigations here, cannot be obtained using these approaches. The remaining options include resorting to dynamical approximations, ${ }^{34,35}$ reduced-dimensionality approaches, ${ }^{36-38}$ or semiclassical methods. ${ }^{39-43}$

The development in this paper belongs to the class of semiclassical methods based on the instanton theory. ${ }^{44-46}$ In the standard instanton formulation, ${ }^{47}$ tunneling splitting is calculated from the zero-temperature limit of the quantum partition function in the path-integral formalism. The dominant contribution to the partition function comes from the minimum action path (MAP) that connects the symmetry-related minima. The contribution from all other paths is estimated analytically using the parameters in a harmonic expansion of the potential in the directions perpendicular to the MAP. Instanton theories of tunneling splittings come in several variants. Some approaches use approximate MAPs, ${ }^{48,49}$ determined from the stationary points on the PES, and approximate Hamiltonians, ${ }^{50,51}$ in which analytic expressions for vibrational couplings are fitted to the PES. The present contribution belongs to the category that is based on the numerically exact MAPs. Mil'nikov and Nakamura $^{52,53}$ used the exact MAP and Hessians along the MAP to obtain splittings via the integration of Jacobi fields (henceforth referred to as the JFI method). They employ internal coordinates in their treatment in order to separate the overall rotational motion. The ring-polymer instanton (RPI) method ${ }^{14,54}$ likewise uses the numerically exact MAP and evaluates the splitting from the eigenvalues of the discretized functional determinant of the action Hessian. This approach is therefore computationally more demanding than the JFI method, ${ }^{52}$ and recovering the rotational dependence of the splittings, when it is significant, becomes elaborate. ${ }^{55}$ Its advantage is that it can be applied without modification to any molecule of interest as it works in Cartesian coordinates, and it can be readily applied to systems that exhibit asymmetric MAPs. The RPI method featured prominently in the recent calculations of tunneling splitting patterns in water clusters. It was used to obtain the ground-state tunneling splitting pattern and reveal mechanisms responsible for its formation in asymmetric systems such as the water dimer, trimer, hexamer, ${ }^{20}$ and octamer ${ }^{56}$ in full dimensionality.

Standard instanton approaches for calculating tunneling splittings suffer from the same drawback as the Monte Carlo and pathintegral based method mentioned above in that they cannot provide the splittings of vibrationally excited states from the outset. It is well known though that the instanton expression for the groundstate tunneling splitting can be obtained using a variant of the WKB theory $^{57}$ and Herring formula. ${ }^{58,59}$ This link thus provides a consistent route for calculating tunneling splittings of vibrationally excited states, ${ }^{52,53,60}$ where this paper aims to contribute. In fact, the semiclassical methods based on the wavefunction along the classical trajectory that connects the minima on the inverted PES, i.e., along the MAP, are regularly referred to as the instanton methods in the literature. ${ }^{53,61,62}$ Tunneling splittings of vibrationally excited states have been obtained using the related methods in symmetric systems such as malonaldehyde, ${ }^{62}$ tropolone, ${ }^{63}$ 9-hydroxyphenalenone, ${ }^{64} \mathrm{HO}_{2},{ }^{53}$ formic acid dimer, ${ }^{65}$ and vinyl radical. ${ }^{6}$

In our recent work, ${ }^{67}$ we generalized the JFI approach of Mil'nikov and Nakamura ${ }^{52}$ to obtain the ground-state tunneling splittings for asymmetric paths in Cartesian coordinates. We obtained an almost perfect agreement between the JFI and RPI splittings ${ }^{67}$ for systems in which rotations do not couple strongly to the internal degrees of freedom, such as water trimer or malonaldehyde. The development enabled us to treat large asymmetric systems that exhibit slow motion of a heavy-atom skeleton, such as the water pentamer, ${ }^{17}$ in full dimensionality. We were able to calculate the 320-level ground-state splitting pattern of the pentamer, including the state symmetries, and to identify rearrangement motions responsible for its formation, in a treatment that would become extremely cumbersome in the RPI approach due to the large imaginary time periods involved.

Motivated by the effectiveness of our JFI approach, the present work aims to derive the tunneling splittings of vibrationally excited states for general symmetric and asymmetric paths in a consistent approach. This is accomplished by a WKB construction of wavefunction that reproduces our JFI result in the ground state. In essence, our approach below follows the work of Mil'nikov and Nakamura ${ }^{53,60}$ in which they extend their ground-state instanton theory of Ref. 52 to treat the low-lying vibrationally excited states. Distinctly, in our approach, we can readily treat asymmetric paths, which are regularly encountered in the studies of clusters, and we again work in Cartesian coordinates in order to make our approach general. Unlike Ref. 53, we treat the "longitudinal" modes, which are parallel to the MAP at minima, and "transversal" modes, which are perpendicular to the MAP at minima, on an equal footing. We achieve this by using a different form of the matching wavefunction near minima, which allows for a displacement of the wavefunction node away from the MAP. In particular, this means that we can treat the asymmetric paths in which the excited mode is the longitudinal mode at one minimum and is a transversal mode near the other end of the MAP. The straightforward generalization of Ref. 53 to asymmetric paths would give a zero splitting in that case. The theory thus includes newly added terms that for certain cases dramatically improve the splitting estimates even in symmetric systems. It is applicable to low vibrationally excited states.

The instanton method evaluates the splittings with a modest number of potential evaluations (on the order of a thousand) in comparison with the exact methods. ${ }^{60,68,69}$ This means that the computations can be performed on larger systems or using more accurate electronic potentials. In certain circumstances, it can probably provide the best possible splittings in a compromise between the accuracy of the dynamical theory and the level of electronic structure 
theory that the dynamical treatment allows. Numerical effort is concentrated in the MAP optimization and the Hessian evaluation along the MAP. ${ }^{68,69}$ Since the calculations of splittings in vibrationally excited states do not require any additional information about the molecular system, they too enjoy the same advantages over the exact methods.

This paper is organized as follows: In Sec. II, we use a semiclassical expansion to approximate the wavefunction about the MAP. The wavefunctions that start from the "left" and from the "right" symmetry-connected minima along the MAP are constructed and used in the Herring formula at the dividing surface to obtain the ground-state tunneling splitting, which is identical in form to the JFI instanton expression from our previous work. ${ }^{67}$ The derivation follows Ref. 52 but does not assume the mirror symmetry of the potential along the MAP. We prove explicitly that the expression for the splitting does not depend on the position of the connection point between the left- and right-localized wavefunctions along the MAP. Section II thus lays the groundwork for constructing the wavefunctions of the excited states in Sec. III. Section III follows the work of Ref. 53 but arrives at a different expression for the tunneling splittings of vibrationally excited states. As stated above, our formulation treats longitudinal and transversal excitations in a unified approach. In certain cases, as the numerical exercises on symmetric and asymmetric model potentials in Sec. IV show, the contribution from the newly added terms can dominate the splittings. The deuterated water dimer provides a real-life test system that exhibits asymmetric paths, including the path featuring the longitudinal-transversal excitation mode and the vibrational modes that do not line up in either parallel or perpendicular direction with respect to the MAP near minima. The importance of different terms in the semiclassical expansion is discussed in terms of the accuracy improvements that they bring to the splittings and the stability with regards to the position of the dividing surface. Conclusions and outlook are given in Sec. V. Atomic units $(\hbar=1)$ are used throughout unless indicated otherwise.

\section{GROUND-STATE TUNNELING SPLITTING}

Tunneling splittings in molecular systems with multiple symmetry-related minima can be expressed as the eigenvalues of a tunneling matrix ${ }^{14}$ in which rows and columns are numbered by the indices of the minima, using group theoretic arguments. The tunneling matrix element $h$ connecting two minima, termed $\mathrm{L}$ and $\mathrm{R}$ for convenience, is the transition amplitude between the degenerate states $\phi^{(\mathrm{L})}$ and $\phi^{(\mathrm{R})}$, localized in their respective wells, which neglect the presence of tunneling motion. The tunneling splitting of the isolated double-well system connecting minima $\mathrm{L}$ and $\mathrm{R}$ is thus $\Delta=-2 h$, the difference between the tunneling matrix eigenvalues. The tunneling matrix eigenvectors are comprised of the coefficients of the energy eigenstates in the $\phi^{(\mathrm{L} / \mathrm{R})}$ basis. For a double-well system, they form the symmetric and antisymmetric linear combinations of $\phi^{(\mathrm{L})}$ and $\phi^{(\mathrm{R})}$.

In our previous work, ${ }^{67}$ we derived the tunneling matrix element $h$, or equivalently the tunneling splitting $\Delta$, using the JFI theory. The splitting is dominated by the Euclidean action of the MAP, while the contributions from all other paths in the harmonic neighborhood of the MAP are collected into the fluctuation prefactor.
The fluctuation prefactor is then evaluated via integration of Jacobi fields. ${ }^{52,70}$ We now proceed along the lines of Refs. 53, 57, and 71 to derive an identical expression using the semiclassical WKB approach to construct the localized states $\phi^{(\mathrm{L} / \mathrm{R})}$.

Whenever the energy eigenstates are well approximated by the symmetric and antisymmetric combinations of the localized state functions, $\phi^{(\mathrm{L} / \mathrm{R})}$, the tunneling splitting can be calculated using the Herring formula, ${ }^{58,59}$

$$
\Delta=\frac{\int\left(\phi^{(\mathrm{L})} \frac{\partial}{\partial S} \phi^{(\mathrm{R})}-\phi^{(\mathrm{R})} \frac{\partial}{\partial S} \phi^{(\mathrm{L})}\right) \delta(f(\mathbf{x})) \mathrm{d} \mathbf{x}}{\int\left|\phi^{(\mathrm{L})}\right|^{2} \mathrm{~d} \mathbf{x}},
$$

where $\mathbf{x}$ is the molecular geometry in mass-scaled Cartesian coordinates and $f(\mathbf{x})=0$ is an implicit equation of an arbitrary dividing plane, which separates the two minima. The variable $S$ corresponds to the position on a local normal to the dividing plane.

We now construct the localized states $\phi^{(\mathrm{L} / \mathrm{R})}$ in the familiar $\mathrm{WKB}$ form as

$$
\phi=\mathrm{e}^{-\frac{1}{\hbar}\left(W_{0}+W_{1} \hbar\right)},
$$

where we drop the labels (L/R) from this point onward as the equations are valid in both wells. In Eq. (2), $W_{0}$ satisfies the HamiltonJacobi equation

$$
\frac{\partial W_{0}}{\partial x_{i}} \frac{\partial W_{0}}{\partial x_{i}}=2 V(\mathbf{x})
$$

where $V(\mathbf{x})$ is the PES, and $W_{1}$ satisfies the transport equation

$$
\frac{\partial W_{0}}{\partial x_{i}} \frac{\partial W_{1}}{\partial x_{i}}-\frac{1}{2} \frac{\partial^{2} W_{0}}{\partial x_{i} \partial x_{i}}+E=0 .
$$

We note here that $E$ is approximated by the ground-state energy of the quantum harmonic oscillator and is of the order $\hbar^{1}$. The whole energy dependence is moved to the transport equation [Eq. (4)] following Refs. 52 and 57.

The Hamilton-Jacobi equation [Eq. (3)] can be solved using the method of characteristics that we briefly describe in Appendix A. The characteristics of the Hamilton-Jacobi equation are given by

$$
\ddot{\mathbf{x}}(\tau)=\nabla V(\mathbf{x}(\tau)),
$$

with $\tau$ as a parameter. The form of Eq. (5) suggests that the characteristics represent classical trajectories on the inverted PES and that $\tau$ represents time. As shown in Appendix A, these trajectories must have zero energy in order to satisfy Eq. (3). On a characteristic, $W_{0}$ can be obtained by a simple integration,

$$
W_{0}\left(\mathrm{x}\left(\tau_{2}\right)\right)=W_{0}\left(\mathrm{x}\left(\tau_{1}\right)\right)+\int_{\tau_{1}}^{\tau_{2}} p_{0}^{2}(\tau) \mathrm{d} \tau,
$$

where $p_{0}=\sqrt{2 V}$ corresponds to the mass-scaled momentum on the classical trajectory. It is convenient to choose one point to correspond to the minimum of the PES and define $W_{0}\left(\mathbf{x}_{\text {min }}\right)=0$. The reason behind this choice is that in the vicinity of the minimum, the wavefunction can then be matched to that of the harmonic oscillator, which will be used later on to determine its norm. With that choice, since the minimum on the PES is a maximum on the inverted PES, all other points along the characteristic correspond to times $\tau>\tau_{\min }$ and the integral in Eq. (6) remains positive. However, by choosing 
the first point at the minimum, the time to any other point will be infinite, since it takes infinite time to move away from the minimum with zero energy. This presents a problem in a numerical implementation, which can conveniently be fixed by reparametrizing the characteristics using the arc length distance $S$ from the minimum along the characteristic,

$$
\frac{\mathrm{d} S}{\mathrm{~d} \tau}=\sqrt{\frac{\mathrm{d} x_{i}}{\mathrm{~d} \tau} \frac{\mathrm{d} x_{i}}{\mathrm{~d} \tau}}=p_{0} .
$$

Using this transformation, Eq. (6) reduces to

$$
W_{0}(\mathbf{x})=\int_{0}^{S(\mathbf{x})} p_{0}\left(S^{\prime}\right) \mathrm{d} S^{\prime}
$$

We observe that $W_{0}$ equals Jacobi action between the minimum and the point $S$ on the characteristic. The characteristic between the minimum and a point $\mathbf{x}$, as well as $W_{0}$, can both be determined by a Jacobi action minimization. The gradient of $W_{0}$ is therefore parallel to the characteristic.

In order to describe $W_{0}$ in the vicinity of a given characteristic, we assume that the Hessian of the potential, $\mathbf{H}(S)$, along the characteristic is known. The equation for the Hessian of $W_{0}, A_{i j}=\frac{\partial^{2} W_{0}}{\partial x_{i} \partial x_{j}}$, along a characteristic is then obtained, by differentiating Eq. (3) twice, as

$$
p_{0} \frac{\partial}{\partial S} \mathbf{A}(S)=\mathbf{H}(S)-\mathbf{A}^{2}(S)
$$

The Riccati equation in Eq. (9) is identical to the equation that emerges in the JFI method ${ }^{52,67}$ as the equation for the log-derivative of a Jacobi field. The initial condition for Eq. (9) at the minimum, where $p_{0}=0$, is $\mathbf{A}_{0}=\mathbf{H}(0)^{1 / 2}$. This identification later serves to match the semiclassical wavefunction $\phi$ in Eq. (2) to that of the harmonic oscillator at the minimum.

We can now expand $W_{0}$ around the characteristic as

$$
W_{0}(S, \Delta \mathbf{x})=\int_{0}^{S} p_{0}\left(S^{\prime}\right) \mathrm{d} S^{\prime}+\frac{1}{2} \Delta \mathbf{x}^{\top} \mathbf{A} \Delta \mathbf{x},
$$

where $\left\{S, \Delta x_{i}\right\}$ is a set of local coordinates ${ }^{53}$ for an arbitrary point $\mathbf{x}$. The coordinate $S$ corresponds to the position of the point $\mathbf{x}_{0}$ on the characteristic that satisfies $\left(x_{i}-x_{0 i}\right) p_{0 i}=0$. The coordinates $\Delta x_{i}$ define an orthogonal shift from $\mathbf{x}_{0}$ to $\mathbf{x}$ so that $\Delta x_{i}=x_{i}-x_{0 i}$. The Jacobian of the transformation is derived in Appendix A. The first term in the expansion is missing since $\nabla W_{0}$ is tangent to the classical trajectory. Equation (10) serves to describe $W_{0}$ in the neighborhood of the characteristic without the need to compute new characteristics.

The transport equation in Eq. (4) can be solved on a characteristic by a simple integration,

$$
W_{1}(S)=\frac{1}{2} \int_{0}^{S} \frac{\operatorname{Tr}\left(\mathbf{A}\left(S^{\prime}\right)-\mathbf{A}_{0}\right)}{p_{0}} \mathrm{~d} S^{\prime}
$$

where we inserted the energy of the harmonic oscillator $E=\frac{1}{2} \operatorname{Tr} \mathbf{A}_{0}$ into the expression. Using Eqs. (10) and (11), the localized wavefunctions in Eq. (2) take the following forms in their respective wells:

$$
\begin{aligned}
& \phi^{(\mathrm{L})}(S)=\mathrm{e}^{-\int_{0}^{S} p_{0}\left(S^{\prime}\right) \mathrm{d} S^{\prime}-\frac{1}{2} \int_{0}^{S} \frac{\operatorname{Tr}\left(\mathbf{A}^{(\mathrm{L})}\left(S^{\prime}\right)-\mathbf{A}_{0}^{(\mathrm{L})}\right)}{p_{0}} \mathrm{~d} S^{\prime}-\frac{1}{2} \Delta \mathbf{x}^{\top} \mathbf{A}^{(\mathrm{L})} \Delta \mathbf{x},} \\
& \phi^{(\mathrm{R})}(\tilde{S})=\mathrm{e}^{-\int_{0}^{\hat{S}} p_{0}\left(\tilde{S}^{\prime}\right) \mathrm{d} \tilde{S}^{\prime}-\frac{1}{2} \int_{0}^{\tilde{S}} \frac{\operatorname{Tr}\left(\mathbf{A}^{(\mathrm{R})}\left(\tilde{S}^{\prime}\right)-\mathbf{A}_{0}^{(\mathrm{R})}\right)}{p_{0}} \mathrm{~d} \tilde{S}^{\prime}-\frac{1}{2} \Delta \mathbf{x}^{\top} \mathbf{A}^{(\mathrm{R})} \Delta \mathbf{x},}
\end{aligned}
$$

where $S$ is the distance from the left minimum along the characteristic, while $\tilde{S}$ denotes the corresponding distance from the right minimum. In the harmonic regions near minima, these wavefunctions are matched to that of the quantum harmonic oscillator, as we describe in Appendix B. From that identification, we obtain their norm as

$$
\int|\phi|^{2} \mathrm{~d} \mathbf{x}=\sqrt{\frac{\pi^{N}}{\operatorname{det} \mathbf{A}_{0}}} .
$$

Having obtained the localized wavefunctions in Eqs. (12) and (13), we are ready to compute the tunneling splitting via the Herring formula in Eq. (1). One could take an arbitrary dividing surface and compute the surface integral in Eq. (1) numerically. However, this requires computing the characteristics that connect the minima with every point at which the integrand is evaluated on the dividing surface. An economical way to compute the integral is to choose one point on the dividing surface and use the Taylor expansion of $W_{0}$ around it to evaluate the integrand at other points. If the dividing surface is chosen to be a hyperplane and the gradient of $W_{0}$ is taken to be constant, the integral can be computed analytically. Since the integrand in the Herring formula is proportional to the product $\phi^{(\mathrm{L})} \phi^{(\mathrm{R})}$, the integral will be best approximated if the point on the dividing surface is chosen so that it maximizes this product. This is equivalent to the minimization of

$$
\int_{0}^{S^{(\mathrm{L})}} p_{0}^{(\mathrm{L})}\left(S^{\prime}\right) \mathrm{d} S^{\prime}+\int_{0}^{\tilde{S}^{(\mathrm{R})}} p_{0}^{(\mathrm{R})}\left(\tilde{S}^{\prime}\right) \mathrm{d} \tilde{S}^{\prime},
$$

which is accomplished when the point lies on the classical trajectory that connects the two minima. In that case, the characteristics that originate at two minima are smoothly joined at the connection point $S=S_{\mathrm{cp}}$ and $\tilde{S}=S_{\text {tot }}-S_{\mathrm{cp}}$, where $S_{\text {tot }}$ is the total length of the MAP that connects the two minima. The two joined characteristics coincide with the instanton trajectory. ${ }^{45,52}$ The sum of $W_{0}^{(\mathrm{L})}$ and $W_{0}^{(\mathrm{R})}$ then becomes the Jacobi action of the instanton trajectory, $W_{0}^{(\mathrm{L})}+W_{0}^{(\mathrm{R})}=\int_{0}^{S_{\text {tot }}} p_{0} \mathrm{~d} S$. The dividing surface is taken to be orthogonal to the trajectory at the connection point, and the Herring formula gives the ground-state tunneling splitting as

$$
\begin{aligned}
\Delta_{0}= & \sqrt{\frac{\operatorname{det} \mathbf{A}_{0}}{\pi^{N}}} \mathrm{e}^{-\int_{0}^{S_{\text {tot }}} p_{0} \mathrm{~d} S-W_{1}^{(\mathrm{L})}-W_{1}^{(\mathrm{R})}} \\
& \times \int\left(\frac{\partial W_{0}^{(\mathrm{L})}}{\partial S}-\frac{\partial W_{0}^{(\mathrm{R})}}{\partial S}\right) \mathrm{e}^{-\Delta \mathbf{x}^{\top} \frac{\mathbf{A}^{(\mathrm{L})}+\mathbf{A}^{(\mathrm{R})}}{2} \Delta \mathbf{x}} \delta(f(\mathbf{x})) \mathrm{d} \mathbf{x},
\end{aligned}
$$

where $\frac{\partial W_{0}^{(\mathrm{R})}}{\partial S}=-\frac{\partial W_{0}^{(\mathrm{R})}}{\partial \tilde{S}}$ evaluates to $p_{0}$ at the connection point and is kept constant in the surface integral.

In order to solve the integral in Eq. (15), we note that the matrix

$$
\overline{\mathbf{A}}=\frac{\mathbf{A}^{(\mathrm{L})}+\mathbf{A}^{(\mathrm{R})}}{2}
$$

possesses a zero eigenvalue, which corresponds to the tangent vector. This is easily proved by differentiating the Hamilton-Jacobi 
equation [Eq. (3)], which yields $\mathbf{A}^{(\mathrm{L} / R)} \mathbf{p}_{0}^{(\mathrm{L} / R)}=\nabla V$. Subtracting these two equations and using the fact that $\mathbf{p}_{0}^{(\mathrm{L})}=-\mathbf{p}_{0}^{(\mathrm{R})}$, it follows that $\left(\mathbf{A}^{(\mathrm{L})}+\mathbf{A}^{(\mathrm{R})}\right) \mathbf{p}_{0}^{(\mathrm{L})}=\mathbf{0}$. The eigenvectors of $\overline{\mathbf{A}}$ that correspond to its non-zero eigenvalues $\lambda_{i}$ then span the dividing surface. Transforming to the eigenvector basis reduces this integral to

$$
\begin{aligned}
\Delta_{0} & =2 p_{0} \sqrt{\frac{\operatorname{det} \mathbf{A}_{0}}{\pi^{N}}} \mathrm{e}^{-\int_{0}^{S_{\text {tot }}} p_{0} \mathrm{~d} S-W_{1}^{(\mathrm{L})}-W_{1}^{(\mathrm{R})}} \int \mathrm{e}^{-\lambda_{i} \xi_{i}^{2}} \mathrm{~d} \xi \\
& =2 p_{0} \sqrt{\frac{\operatorname{det} \mathbf{A}_{0}}{\pi \operatorname{det}^{\prime} \overline{\mathbf{A}}}} \mathrm{e}^{-\int_{0}^{S_{\text {tot }}} p_{0} \mathrm{~d} S-W_{1}^{(\mathrm{L})}-W_{1}^{(\mathrm{R})}},
\end{aligned}
$$

where $\operatorname{det}^{\prime}$ denotes the product of non-zero $\lambda_{i}$ 's, and $W_{1}^{(\mathrm{L} / \mathrm{R})}$ at $S=$ $S_{\mathrm{cp}}$ are calculated using Eq. (11). The ground-state tunneling splitting formula in Eq. (17) is identical to the instanton formula in Eq. (33) of Ref. 67. The splitting in Eq. (17) does not depend on the position of the connection point on the instanton trajectory. This is evident from the derivation of Ref. 67, but the present treatment does not guarantee it, and we prove it in Appendix D.

\section{EXCITED-STATE TUNNELING SPLITTING}

The calculation of tunneling splittings in vibrationally excited states is approached in a consistent manner, following Ref. 53. We assume one quantum of vibrational excitation in the mode with frequency $\omega_{\mathrm{e}}$ and construct the WKB wavefunctions in Eq. (2) by solving the Hamilton-Jacobi and transport equations [Eqs. (3) and (4)] and finally insert them into the Herring formula [Eq. (1)], which remains valid for the excited states.

Only the transport equation depends on the energy and is different for the excited state. We decompose $W_{1}$ in the form

$$
W_{1}=W_{1}^{(0)}+w
$$

where $W_{1}^{(0)}$ is the ground-state function given by Eq. (11), and insert Eq. (18) in Eq. (4). We then find that $w$ satisfies

$$
\frac{\partial W_{0}}{\partial x_{i}} \frac{\partial w}{\partial x_{i}}+\omega_{\mathrm{e}}=0
$$

In a crucial difference from Ref. 53, we seek the solution of Eq. (19) along the characteristic in the following form:

$$
w=-\ln \left(\mathbf{U}^{\top} \Delta \mathbf{x}+F\right) .
$$

The above form, when used in Eq. (2), allows the matching to a harmonic oscillator wavefunction in the neighborhood of minima for both the longitudinally and transversally excited modes with respect to the MAP in a unified approach. We insert Eq. (20) into Eq. (19), multiply through with $\mathbf{U}^{\top} \Delta \mathbf{x}+F$, and equate the terms of order $\Delta \mathbf{x}^{0}$ and $\Delta \mathbf{x}^{1}$ to obtain equations for $F$ and $\mathbf{U}$ as

$$
\begin{gathered}
p_{0} \frac{\mathrm{d}}{\mathrm{d} S} F=\omega_{\mathrm{e}} F, \\
p_{0} \frac{\mathrm{d}}{\mathrm{d} S} \mathbf{U}=\omega_{\mathrm{e}} \mathbf{U}-\mathbf{A} \mathbf{U}+2\left(\mathbf{U}^{\top} \mathbf{p}_{0}-\omega_{\mathrm{e}} F\right) \frac{\mathbf{A} \mathbf{p}_{0}}{p_{0}^{2}} .
\end{gathered}
$$

Equation (22) can be simplified by noting that, by definition, components of $\mathbf{U}$ equal to

$$
U_{i}=\frac{\partial}{\partial x_{i}} \mathrm{e}^{-w}=\frac{\partial}{\partial x_{i}} F
$$

where the second equality is due to the fact that the partial derivative is taken on the characteristic. This means that the projection of $\mathbf{U}$ onto the tangent is

$$
\mathbf{U}^{\top} \mathbf{p}_{0}=\frac{\partial F}{\partial x_{i}} \frac{\partial W_{0}}{\partial x_{i}}=p_{0} \frac{\mathrm{d}}{\mathrm{d} S} F=\omega_{\mathrm{e}} F,
$$

where Eq. (21) was used. Combining Eqs. (22) and (24) reduces the equation for $\mathbf{U}$ to

$$
p_{0} \frac{\mathrm{d}}{\mathrm{d} S} \mathbf{U}=\omega_{\mathrm{e}} \mathbf{U}-\mathbf{A} \mathbf{U} .
$$

This is the same equation that Mil'nikov and Nakamura ${ }^{53}$ obtained in their treatment of transversal excitations. Here, however, we use it for both longitudinal and transversal excitations. As our test calculations below demonstrate, it is important to propagate both components of $\mathbf{U}$ simultaneously for best accuracy.

Equations (21) and (25) have singularities at the minima of PES. In order to avoid them, we need to start the propagation a small distance $\varepsilon$ away from the minimum along the characteristic. If this distance is sufficiently small to fall into the harmonic region around the minimum, the initial conditions at $\varepsilon$ can be taken in the form

$$
F(\varepsilon)=\mathbf{U}_{0}^{\top}\left(\mathbf{x}_{0}(\varepsilon)-\mathbf{x}_{0}(0)\right),
$$

as justified in Appendix B, and

$$
\mathbf{U}(\varepsilon)=\mathbf{U}_{0},
$$

where $\mathbf{U}_{0}$ is the excited normal mode at the minimum.

Alternatively, we can solve Eq. (25) in the region $[0, \varepsilon]$ using the same procedure that was used for solving Eq. (9) in Refs. 52 and 67. We expand $p_{0}, \mathbf{A}$, and $\mathbf{U}$ around minimum as

$$
\begin{aligned}
p_{0} & =p_{0}^{(1)} S, \\
\mathbf{A} & =\mathbf{A}_{0}+\mathbf{A}_{1} S, \\
\mathbf{U} & =\sum_{i} \mathbf{C}^{(i)} S^{i} .
\end{aligned}
$$

We then insert Eq. (28) into Eq. (25) and equate the terms of the same order in $S^{i}$ to obtain the recurrence relation for $\mathbf{C}^{(i)}$,

$$
\begin{aligned}
\mathbf{A}_{0} \mathbf{C}^{(0)} & =\omega_{\mathrm{e}} \mathbf{C}^{(0)}, \\
\left(\mathbf{A}_{0}+\left(i p_{0}^{(1)}-\omega_{\mathrm{e}}\right) \mathbf{I}\right) \mathbf{C}^{(i)} & =-\mathbf{A}_{1} \mathbf{C}^{(i-1)} .
\end{aligned}
$$

Once $\mathbf{U}$ has been determined, $F$ can be obtained from Eq. (24) as

$$
F(S)=\int_{0}^{S} \mathbf{U}^{\top}\left(S^{\prime}\right) \mathbf{t}\left(S^{\prime}\right) \mathrm{d} S^{\prime}=\frac{\mathbf{U}^{\top}(S) \mathbf{p}_{0}(S)}{\omega_{\mathrm{e}}},
$$

where $\mathbf{t}=\mathbf{p}_{0} / p_{0}$ is the tangent vector at the instanton trajectory. In this way, the anharmonicity of the PES near minima is accounted for by $\mathbf{A}_{1}$. Having obtained $\mathbf{U}(\varepsilon)$, Eq. (25) is readily solved by a simple integrator, such as the Runge-Kutta method.

At the dividing plane, the wavefunction of the excited state in Eq. (2) takes the form 


$$
\begin{aligned}
& \phi^{(\mathrm{L})}=\left(\mathbf{U}^{(\mathrm{L}) \top} \Delta \mathbf{x}+F^{(\mathrm{L})}\right) \mathrm{e}^{-\int_{0}^{S} p_{0}\left(S^{\prime}\right) \mathrm{d} S^{\prime}-\frac{1}{2} \int_{0}^{S} \frac{\operatorname{Tr}\left(\mathbf{A}^{(\mathrm{L})}\left(S^{\prime}\right)-\mathbf{A}_{0}^{(\mathrm{L})}\right)}{p_{0}} \mathrm{~d} S^{\prime}-\frac{1}{2} \Delta \mathbf{x}^{\top} \mathbf{A}^{(\mathrm{L})} \Delta \mathbf{x},} \\
& \phi^{(\mathrm{R})}=\left(\mathbf{U}^{(\mathrm{R}) \top} \Delta \mathbf{x}+F^{(\mathrm{R})}\right) \mathrm{e}^{-\int_{0}^{\tilde{S}} p_{0}\left(\tilde{S}^{\prime}\right) \mathrm{d} \tilde{S}^{\prime}-\frac{1}{2} \int_{0}^{\tilde{S}} \frac{\operatorname{Tr}\left(\mathbf{A}^{(\mathrm{R})}\left(\tilde{S}^{\prime}\right)-\mathbf{A}_{0}^{(\mathrm{R})}\right)}{p_{0}} \mathrm{~d} \tilde{S}^{\prime}-\frac{1}{2} \Delta \mathbf{x}^{\top} \mathbf{A}^{(\mathrm{R})} \Delta \mathbf{x}} .
\end{aligned}
$$

By matching the above wavefunction to that of the harmonic oscillator at a minimum, one obtains the norm as

$$
\int|\phi|^{2} \mathrm{~d} \mathbf{x}=\sqrt{\frac{\pi^{N}}{\operatorname{det} \mathbf{A}_{0}}} \frac{1}{2 \omega_{\mathrm{e}}} .
$$

The wavefunctions in Eq. (31) are then inserted into the Herring formula, and the surface integral is evaluated in a similar manner to the ground-state case. This gives the tunneling splitting of vibrationally excited states as

$$
\Delta_{1}=\Delta_{0}\left(2 \omega_{\mathrm{e}}\right)\left(F^{(\mathrm{L})} F^{(\mathrm{R})}+\frac{1}{2} \mathrm{U}^{(\mathrm{L})} \bar{A}^{-1} \mathrm{U}^{(\mathrm{R})}\right) .
$$

Since $\overline{\mathbf{A}}$ possesses a zero eigenvalue, $\overline{\mathbf{A}}^{-1}$ in Eq. (33) denotes a pseudoinverse of $\overline{\mathbf{A}}$, defined by $\overline{\mathbf{A}} \overline{\mathbf{A}}^{-1}=\overline{\mathbf{A}}^{-1} \overline{\mathbf{A}}=\mathbf{P}$, where $\mathbf{P}=\mathbf{I}-\mathbf{t t}^{\top}$ is a projector onto the orthogonal plane. The pseudoinverse has the same eigenvectors as $\overline{\mathbf{A}}$, while its nonzero eigenvalues are reciprocals of the eigenvalues of $\overline{\mathbf{A}}$.

It turns out that the tunneling splitting formula in Eq. (33) is dependent on the position of the connection point at which the dividing surface and the instanton trajectory cross. This undesirable behavior, which was not present in the ground-state formula in Eq. (17), arises from the $\mathbf{U}^{(\mathrm{L})} \overline{\mathbf{A}}^{-1} \mathbf{U}^{(\mathrm{R})}$ term, as shown in Appendix $D$. It can further be shown, by a similar analysis, that the terms that cause this dependency cancel out if the next order term is included in the Taylor expansion of $\exp (-w)$,

$$
w=-\ln \left(F+U_{i} \Delta x_{i}+\frac{1}{2} Z_{i j} \Delta x_{i} \Delta x_{j}\right) .
$$

However, the inclusion of $\mathbf{Z}$ in Eq. (34) brings new terms that again do depend on the connection point, and to eliminate their dependence on $S_{\mathrm{cp}}$, it would be necessary to include higher order terms in the wavefunction expansion in Eq. (2), such as the $W_{2}$ term. The root of the problem is that the expansion of $\exp (-w)$ is inconsistent with the expansion of $W_{1}$ as it gives rise to terms of all orders in $\Delta \mathbf{x}$ in the expansion of $w$. Excluding the higher order terms of $w$ in Eq. (34), on the other hand, would degrade the quality of matching with the harmonic oscillator near minima.

In fact, any improvement of the accuracy of the WKB wavefunction through the inclusion of extra terms in $W_{0}$ and $W_{1}$ necessarily requires the calculation of higher order derivatives of potential along the path. Calculation of the tensor of third derivatives of potential along the path allows us to expand $W_{0}$ in Eq. (10) up to the $\Delta \mathbf{x}^{3}$ term and $W_{1}^{(0)}$ in Eq. (11) up to $\Delta \mathbf{x}^{1}$, and to include the $\Delta \mathbf{x}^{2}$ term in Eq. (34). The tensor of fourth derivatives of potential allows for the correction of the vibrational energy and the inclusion of the $\Delta \mathbf{x}^{0}$ term of $W_{2}$ and the higher order terms in $W_{0}, W_{1}^{(0)}$, and $w$. The calculation of higher order derivatives of the potential quickly becomes computationally unfeasible for realistic potential energy functions and, in most cases, does not improve the results significantly.

In order to study the effect of anharmonicity that originates from the inclusion of third derivatives of potential on the tunneling splittings in numerical tests below, we derive the equation for $\mathbf{Z}$ along a characteristic in Appendix C. It turns out that from all terms that can be computed using the third derivatives of potential, this is the only term that is meaningful to include in the tunneling splitting formula of Eq. (C14). The inclusion of $\nabla W_{1}^{(0)}$ does not appreciably influence the results, whereas the inclusion of the $\Delta \mathbf{x}^{3}$ term in $W_{0}$ in Eq. (10) does not result in convergent integrals on the dividing surface.

It can be shown, by using the $\mathbf{Z}$ contribution to the splitting, derived in Appendix $\mathrm{C}$, that the connection point is best placed in the middle of the instanton path for symmetric systems, i.e., at the top of the barrier, because, at this place, the $\mathbf{Z}$ contribution is the smallest. We found no such justification for the placement of the connection point in asymmetric systems, so the safest place to set it is at the barrier maximum as well.

Alternatively, we can discard the terms that are responsible for the connection point dependence of the splittings in order to obtain an unambiguous formulation. For this purpose, we decompose the vector $\mathbf{U}$ into longitudinal and transversal parts as

$$
\mathbf{U}=\mathbf{U}_{\perp}+F^{\prime} \mathbf{t}
$$

where $\mathbf{U}_{\perp}$ is the component of $\mathbf{U}$ that is perpendicular to the path. Since only the $\mathbf{U}_{\perp}$ components contribute to the splitting in the $\mathbf{U}^{(\mathrm{L})} \overline{\mathbf{A}}^{-1} \mathbf{U}^{(\mathrm{R})}$ term in Eq. (33), due to the fact that the tangent vector is an eigenvector of $\overline{\mathbf{A}}^{-1}$ with zero eigenvalue, it can be used instead of the vector $\mathbf{U}$. We carry out the separation in Eq. (35) at $S=\varepsilon$ and propagate $\mathbf{U}_{\perp}$ and $F$ independently toward the connection point from both minima. It can be shown that $\mathbf{U}_{\perp}$ satisfies the following equation:

$$
p_{0} \frac{\mathrm{d}}{\mathrm{d} S} \mathbf{U}_{\perp}=\omega_{\mathrm{e}} \mathbf{U}_{\perp}-\mathbf{A} \mathbf{U}_{\perp}-2 \omega_{\mathrm{e}} F \frac{\mathrm{d} \mathbf{t}}{\mathrm{d} S} .
$$

If we neglect the last term in Eq. (36), $\mathbf{U}_{\perp}$ satisfies the same equation as $\mathbf{U}$. The vector $\mathbf{U}_{\perp}$ remains perpendicular to the instanton path, when it is propagated using Eq. (25), and as shown in Appendix D, the splitting becomes independent of the position of the connection point. Since the neglected term is proportional to the curvature of the instanton path, it can safely be neglected for paths with small curvatures. For paths with a large curvature, it turns out (see Sec. IV) that it is better to work with the full vector $\mathbf{U}$ as the deviations in the splittings, when the connection point is moved along the instanton path, are smaller than the error introduced by the above approximation.

\section{NUMERICAL TESTS}

We now perform tests of the above theory on a twodimensional (2D) symmetric system, a 2D asymmetric system, and the deuterated water dimer. Each calculation of the tunneling splitting in a vibrationally excited state is preceded by a calculation of the ground-state tunneling splitting using the JFI method of Ref. 67. 
A JFI calculation starts by an action minimization, using the string or quadratic string method, ${ }^{68,69}$ followed by the evaluation of Hessians along the MAP, and finally, it ends with the computation of $\mathbf{A}$ by solving the Riccati equation in Eq. (9) along the MAP. Excitedstate calculations additionally require a propagation of $\mathbf{U}$ along the MAP using Eq. (25) for each vibrationally excited state of interest. In our tests below, we also evaluate $\mathbf{Z}$ along the MAP in order to check the accuracy and convergence of the obtained results. To accomplish this, we first compute the tensor of third derivatives of potential along the MAP, we then use it to propagate Eq. (C2), and finally, we use $\mathbf{B}$, as well as $\mathbf{A}$ and $\mathbf{U}$, to propagate $\mathbf{Z}$ along the MAP using Eq. (C11). The splittings are evaluated using Eqs. (17), (33), and $(\mathrm{C} 14)$.

In the tests, we discretized all instanton paths using 600 equally spaced beads (or points) in mass-scaled Cartesian coordinates and used the string method of Ref. 68 for the optimization of MAP. In the tests on water dimer, the orientations of end beads were adjusted during optimization by minimizing the distance to the first neighbor bead at every iteration. ${ }^{68}$ The convergence criterion was taken to be the maximum value of the action gradient orthogonal to the string as $\max \left\{S_{i}^{\perp}\right\}<10^{-8}$ a.u.. A large number of beads and a tight convergence criterion were used to ensure that the results do not depend on the accuracy of the MAP. Hessians and third-derivative tensors were computed at all beads using the fourth-order finite difference method with the grid spacing of $10^{-3}$ a.u. In water dimer calculations, we projected out the overall translations and rotations, as described in Ref. 73. Molecular geometries, potential, Hessian matrix elements, and third derivative tensor elements were all interpolated with respect to the mass-scaled arc length distance $S$ along the MAP using natural cubic splines. Equations (17), (33), and (C14) were solved on the interval $[0, \varepsilon]$ by linearization, as described previously in Refs. 52 and 67 and in Appendix C, while on the interval $\left[\varepsilon, S_{\mathrm{cp}}\right]$, they were integrated using the Runge-Kutta method ${ }^{72}$ with the fixed step length of $10^{-3} \mathrm{me}_{\mathrm{e}}^{1 / 2} a_{0}$. The parameter $\varepsilon$ was taken as $\varepsilon=0.1 m_{\mathrm{e}}^{1 / 2} a_{0}$ in all test systems.

The normal modes were calculated at one minimum and obtained at the other minimum by utilizing the symmetry operation that connects them in order to avoid sign ambiguity. Equation (25) was then solved on the interval $[0, \varepsilon]$ using the recurrence relation of Eq. (29). The Taylor series of $\mathbf{U}$ in Eq. (28) was cut when the change in the norm of $\mathbf{U}(\varepsilon)$ fell below the threshold value of $10^{-12}$. On the interval $\left[\varepsilon, S_{\mathrm{cp}}\right]$, we used the exponential propagator to solve Eq. (25),

$$
\mathbf{U}(S+h)=\mathrm{e}^{\left(\omega_{\mathrm{e}} \mathbf{I}-\mathbf{A}\right) \frac{h}{p_{0}}} \mathbf{U}(S),
$$

with a fixed step length $h=10^{-3} \mathrm{me}_{\mathrm{e}}^{1 / 2} a_{0} . F$ values were computed from the tangent projection of the $\mathbf{U}$ vector using Eq. (24). That procedure was found to be less sensitive to the value of $F(\varepsilon)$ than the direct integration of Eq. (21) in Eq. (39).

\section{A. Symmetric double-well 2D potential}

We first test the theory on a model 2D double-well system. We call the system symmetric since the potential along the MAP connecting two minima has a left-right mirror symmetry with the maximum of the potential in the middle of the path. The potential is given by the following equations:

$$
\begin{aligned}
& V(\mathbf{x})=\frac{V_{1} V_{2}}{V_{1}+V_{2}}, \\
& V_{1}(\mathbf{x})=\frac{1}{2}\left(\mathbf{x}-\mathbf{x}^{(1)}\right)^{\top} \mathbf{U}_{1}\left(\begin{array}{cc}
\alpha_{1}^{2} & 0 \\
0 & \alpha_{2}^{2}
\end{array}\right) \mathbf{U}_{1}^{\top}\left(\mathbf{x}-\mathbf{x}^{(1)}\right), \\
& V_{2}(\mathbf{x})=\frac{1}{2}\left(\mathbf{x}-\mathbf{x}^{(2)}\right)^{\top} \mathbf{U}_{2}\left(\begin{array}{cc}
\alpha_{1}^{2} & 0 \\
0 & \alpha_{2}^{2}
\end{array}\right) \mathbf{U}_{2}^{\top}\left(\mathbf{x}-\mathbf{x}^{(2)}\right) \text {, } \\
& \mathbf{U}_{1}=\left(\begin{array}{cc}
\cos \theta & -\sin \theta \\
\sin \theta & \cos \theta
\end{array}\right) \text {, } \\
& \mathbf{U}_{2}=\left(\begin{array}{cc}
-\cos \theta & \sin \theta \\
\sin \theta & \cos \theta
\end{array}\right), \\
& \mathbf{x}^{(1,2)}=(0, \pm \beta)^{\top},
\end{aligned}
$$

where $\mathbf{x}$ are not mass scaled. It has two minima, located at $\mathbf{x}^{(1,2)}$, with normal modes given by matrices $\mathbf{U}_{1,2}$. The parameters were set to $\beta$ $=2, \alpha_{1}=1.265, \alpha_{2}=2$, and $m=27$. Changing the angle $\theta$ changes the angle between the normal modes of the two minima, as shown in Fig. 1. With $\theta=0$, the instanton path is a straight line that connects the two minima, and near minima, the path direction coincides with the lowest normal mode. As values of $\theta$ increase and normal modes rotate, the instanton path does not rotate as quickly near minima. Instead, it picks up a non-zero displacement along the higher normal mode. It turns out that this small displacement can significantly affect the splitting. Combining Eqs. (21) and (26), we obtain $F$ at the dividing plane in the form

$$
F\left(S_{\mathrm{cp}}\right)=\mathbf{U}^{\top}(\mathbf{x}(\varepsilon)-\mathbf{x}(0)) \mathrm{e}^{\omega_{\mathrm{e}} \int_{\varepsilon}^{S_{\mathrm{cp}}} \frac{1}{p_{0}} d S^{\prime}} .
$$

The exponential growth of the $F$ term in Eq. (39) is responsible for this behavior. Even small displacements along the excited mode near

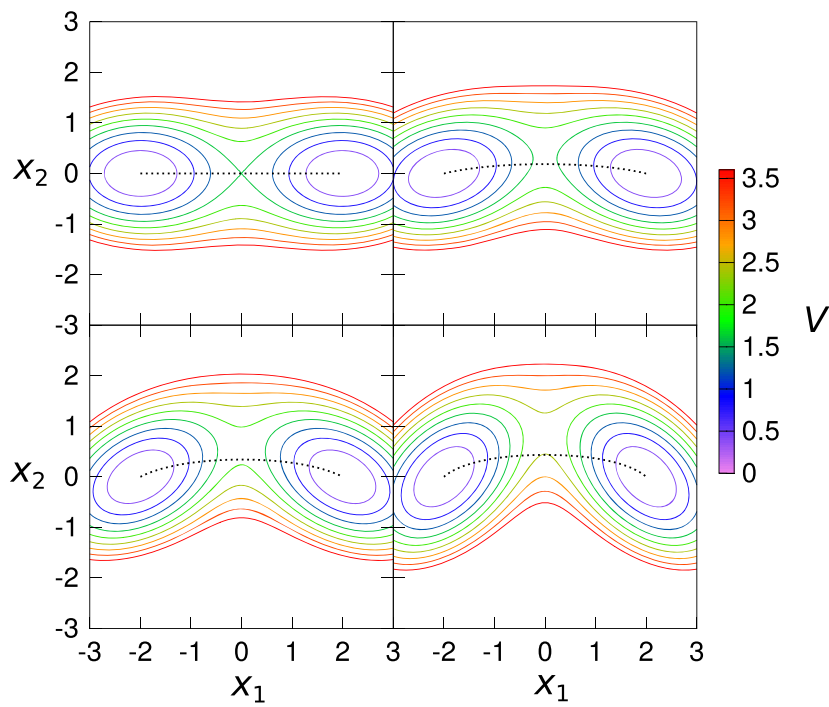

FIG. 1. Potential energy surfaces for the model potential in Eq. (38) $\left(\alpha_{1}=1.265\right.$ $\alpha_{2}=2, \beta=2$ ) for angles $\theta$ of, left to right and top to bottom, $0, \pi / 12, \pi / 6$, and $\pi / 4$. Superposed on each potential energy surface are the corresponding instanton pathways. 
TABLE I. Displacement $\eta$ in Eq. (40) at $\varepsilon=0.1$ for the two normal modes $(1,0)$ and $(0,1)$ of the $2 \mathrm{D}$ symmetric potential in Eq. (38). The fractional contribution of the $F^{(L)} F^{(R)}$ term to the tunneling splitting in Eq. (33), when the mode is excited, is given in parentheses.

\begin{tabular}{lcc}
\hline \hline$\theta$ & $\eta(1,0)$ & $\eta(0,1)$ \\
\hline 0 & $1.00000(1.000)$ & $0.00000(0.000)$ \\
$\pi / 12$ & $0.99998(0.999)$ & $0.00664(0.681)$ \\
$\pi / 6$ & $0.99989(0.995)$ & $0.01488(0.919)$ \\
$\pi / 4$ & $0.99962(0.987)$ & $0.02774(0.976)$ \\
\hline \hline
\end{tabular}

minima can be magnified and result in an important contribution to the splitting. A useful parameter for quantifying the displacement near minima is

$$
\eta=\mathbf{U}_{0}^{\top}(\mathbf{x}(\varepsilon)-\mathbf{x}(0)) / \varepsilon,
$$

where the division with $\varepsilon$ is made to cancel out the dependence on the step length $\varepsilon$, where it is observed. The dependence of the displacement $\eta$ on the angle $\theta$ is given in Table I. It can be seen that the displacement is predominantly along the lower mode for all angles $\theta$ in Table I.

Table II shows the tunneling splittings in the ground state and in the first two excited states, with the lower $(1,0)$ and the higher $(0,1)$ mode excited with one quantum of vibration. The convergence of the excited-state splittings with the addition of $F, \mathbf{U}$, and $\mathbf{Z}$

TABLE II. Tunneling splittings in the ground and first two vibrationally excited states for the potential in Eq. (38) at various angles $\theta$ obtained using instanton theory. The excited-state splittings are, top to bottom, obtained using the expansion of $\exp (-w)$ to $F, F+U_{i} \Delta x_{i}$, and $F+U_{i} \Delta x_{i}+\frac{1}{2} Z_{i j} \Delta x_{i} \Delta x_{j}$ terms. The exact quantum-mechanical results are given in parentheses.

\begin{tabular}{lccc}
\hline \hline$\theta$ & $\Delta_{0}$ & $\Delta_{1}(1,0)$ & $\Delta_{1}(0,1)$ \\
\hline 0 & & $1.830(-8)$ & 0.000 \\
& $2.630(-10)$ & $1.830(-8)$ & $5.026(-10)$ \\
& $(2.639(-10))$ & $1.838(-8)$ & $5.026(-10)$ \\
& & $(1.811(-8))$ & $(5.155(-10))$ \\
$\pi / 12$ & & $9.870(-9)$ & $5.492(-10)$ \\
& $1.463(-10)$ & $9.882(-9)$ & $8.066(-10)$ \\
& $(1.472(-10))$ & $9.927(-9)$ & $8.062(-10)$ \\
& & $(9.858(-9))$ & $(8.089(-10))$ \\
$\pi / 6$ & & $1.563(-9)$ & $4.029(-10)$ \\
& & $1.571(-9)$ & $4.383(-10)$ \\
& $(2.573(-11)$ & $1.578(-9)$ & $4.390(-10)$ \\
& & $(1.583(-9))$ & $(4.477(-10))$ \\
\hline$\pi / 4$ & & $7.729(-11)$ & $5.932(-11)$ \\
& & $7.827(-11)$ & $6.077(-11)$ \\
& & $7.863(-11)$ & $6.097(-11)$ \\
& $(1.620(-12))$ & $(7.879(-11))$ & $(6.224(-11))$ \\
\hline \hline
\end{tabular}

terms in the $\exp (-w)$ expansion is also shown. The exact quantummechanical results are obtained by the diagonalization of Hamiltonian in the sine DVR basis ${ }^{74}$ with grid boundaries at $[-6.0,6.0]$ in both coordinates and 150 basis functions for each degree of freedom. They are given in Table II in parentheses for comparison. It can be seen that the $\mathbf{Z}$ term contribution is small for all the test cases. The contribution of the $F$ term is dominant for the longitudinal excitation of the mode $(1,0)$. On the other hand, when the higher mode $(0$, 1 ) is excited, the relative contribution of $F$ and $\mathbf{U}$ terms changes with angle $\theta$. Displacement $\eta$ suggests that the excitation of $(0,1)$ is in the transversal mode. Indeed, at $\theta=0$, the $F$ term does not contribute and the $\mathbf{U}$ term determines the splitting, as in the theory of Ref. 53. However, with an increase in $\theta$, the $F$ contribution quickly rises to account for more than $90 \%$ of the splitting at $\theta=\pi / 6$, while the displacement remains small at $\eta=0.015$. This demonstrates that it is crucial to include the $F$ term in the expansion of $\exp (-w)$ even when the excited mode appears to be transversal. The contribution from a small displacement can exponentially grow and finally dominate the splitting.

The tunneling splittings are invariant with respect to the position of the dividing plane when only $F$ terms are considered, in accord with the analysis of Appendix D. The same is true for the splitting obtained with the inclusion of the $\mathbf{U}$ terms at $\theta=0$. In this case, the instanton path is a straight line and the vector $\mathbf{U}$ remains perpendicular to the path. We can see that in Eq. (36), the last term disappears in that case since the path curvature is zero. However, we observed in all other cases that the splittings decrease as the position of the dividing plane changes from $0.5 S_{\text {tot }}$ to $0.25 S_{\text {tot }}$. This decrease varies from $0.02 \%$ to $0.2 \%$ for the excitation in the lower, longitudinal, mode and from $3 \%$ to $2 \%$ for the excitation in the higher, transversal, mode. This variation can be eliminated by using $\mathbf{U}_{\perp}$ instead of $\mathbf{U}$, in other words, by ignoring the last term in Eq. (36). In this approach, the $F$ term is still included, e.g., by using Eq. (39), while the $\mathbf{U}^{(\mathrm{L})} \overline{\mathbf{A}}^{-1} \mathbf{U}^{(\mathrm{R})}$ contribution in Eq. (33) is computed with $\mathbf{U}_{\perp}$. This approach thus eliminates the dependence of the splitting on the position of the dividing plane, as discussed in Appendix D. However, we noticed an increase in all computed splittings by as much as $8 \%$, which resulted in an overestimation of quantum-mechanical results. Since the error introduced is larger than the variation of splitting with the connection point position, using the full expression seems to be the preferable option.

In Table III, we studied the dependence of splittings on the reduction in the mass of the system. The convergence of the excitedstate splittings with the addition of $F, \mathbf{U}$, and $\mathbf{Z}$ terms in the $\exp (-w)$ expansion is again shown, as well as the exact quantum-mechanical results in parentheses. The reduction in mass causes an increase in the energy of vibrational states, which provides an insight into the limits of theory as the energy approaches the barrier height. In the ground state, the effective barrier height can be computed as

$$
V_{\mathrm{eff}}^{(0,0)}=V_{0}+\frac{1}{2}\left(\lambda_{2}-\omega_{1}-\omega_{2}\right),
$$

where $V_{0}$ is the potential energy and $\lambda_{2}$ is the nonnegative eigenvalue of matrix $\mathbf{A}$ at the position of the barrier, whereas $\omega_{1}$ and $\omega_{2}$ are vibrational frequencies at the minimum. If the lower, longitudinal mode is excited, the effective barrier is lowered by $\omega_{1}$ and becomes

$$
V_{\mathrm{eff}}^{(1,0)}=V_{\mathrm{eff}}^{(0,0)}-\omega_{1},
$$


TABLE III. Tunneling splittings in the ground $\left(\Delta_{0}\right)$ and first two vibrationally excited states $\left(\Delta_{1}\right)$ for the potential in Eq. (38) at $\theta=\pi / 12$ and various masses $m$ obtained using instanton theory. The excited-state splittings are, top to bottom, obtained using the expansion of $\exp (-w)$ to $F, F+U_{i} \Delta x_{i}$, and $F+U_{i} \Delta x_{i}+\frac{1}{2} Z_{i j} \Delta x_{i} \Delta x_{j}$ terms, respectively. The exact quantum-mechanical results are given in parentheses. For each excitation, the effective barrier heights $V_{\text {eff }}$ on the instanton path are also given.

\begin{tabular}{|c|c|c|c|c|c|}
\hline$m$ & $\Delta_{0}$ & $\Delta_{1}(1,0)$ & $V_{\mathrm{eff}}^{(1,0)}$ & $\Delta_{1}(0,1)$ & $V_{\mathrm{eff}}^{(0,1)}$ \\
\hline 27.0 & $\begin{array}{c}1.463(-10) \\
(1.472(-10))\end{array}$ & $\begin{array}{c}9.870(-9) \\
9.882(-9) \\
9.927(-9) \\
(9.858(-9))\end{array}$ & 1.273 & $\begin{array}{c}5.492(-10) \\
8.066(-10) \\
8.062(-10) \\
(8.089(-10))\end{array}$ & 1.428 \\
\hline 5.0 & $\begin{array}{c}1.431(-4) \\
(1.435(-4))\end{array}$ & $\begin{array}{c}4.156(-3) \\
4.168(-3) \\
4.212(-3) \\
(3.921(-3))\end{array}$ & 0.731 & $\begin{array}{c}2.312(-4) \\
4.831(-4) \\
4.827(-4) \\
(4.979(-4))\end{array}$ & 1.091 \\
\hline 1.7 & $\begin{array}{c}1.264(-2) \\
(1.231(-2))\end{array}$ & $\begin{array}{c}0.214 \\
0.215 \\
0.219 \\
(0.146)\end{array}$ & 0.051 & $\begin{array}{c}1.191(-2) \\
3.416(-2) \\
3.413(-2) \\
(3.080(-2))\end{array}$ & 0.668 \\
\hline 1.5 & $\begin{array}{c}1.865(-2) \\
(1.802(-2))\end{array}$ & $\begin{array}{c}0.297 \\
0.298 \\
0.304 \\
(0.188)\end{array}$ & -0.055 & $\begin{array}{c}1.649(-2) \\
4.932(-2) \\
4.927(-2) \\
(4.157(-2))\end{array}$ & 0.603 \\
\hline 1.0 & $\begin{array}{c}5.696(-2) \\
(5.300(-2))\end{array}$ & $\begin{array}{c}0.740 \\
0.745 \\
0.763 \\
(0.361)\end{array}$ & -0.445 & $\begin{array}{c}0.041 \\
0.141 \\
0.141 \\
(0.102)\end{array}$ & 0.360 \\
\hline
\end{tabular}

while if the higher, transversal mode is excited, the effective barrier changes as

$$
V_{\mathrm{eff}}^{(0,1)}=V_{\mathrm{eff}}^{(0,0)}-\omega_{2}+\lambda_{2} .
$$

As we reduce the effective barrier height, by varying the mass in Table III, the instanton method starts to overestimate the tunneling splittings. When $V_{\text {eff }} \approx 0$, the excited-state splitting is overestimated by about a factor of 2 , similar to the earlier observations in the ground state. ${ }^{54}$ This is mainly caused by the overestimation of the state energy in the harmonic approximation, which is then used in the transport equation. Furthermore, a significant effect comes from the underestimation of the norm of the localized wavefunction in the harmonic approximation as it extends further on the other side of the barrier. Therefore, in the case of a "shallow" splitting or the "over-the-barrier" splitting, the estimates obtained using the instanton method should only serve as an upper limit.

\section{B. Asymmetric double-well 2D potential}

We next perform tests on an asymmetric model $2 \mathrm{D}$ system. The potential profile along the MAP connecting any two minima does not have the left-right symmetry, and the maximum does not, in general, lie at the midpoint. The MAP can approach two minima along different normal modes in an asymmetric system. The asymmetric potential that we use in our tests is given by the following equations:

$$
\begin{aligned}
& V_{1}=\frac{1}{2} \alpha_{1}^{2}\left(x_{1}+\beta\right)^{2}+\frac{1}{2} \alpha_{2}^{2}\left(x_{2}+\beta\right)^{2}, \\
& V_{2}=\frac{1}{2} \alpha_{2}^{2}\left(x_{1}-\beta\right)^{2}+\frac{1}{2} \alpha_{1}^{2}\left(x_{2}+\beta\right)^{2}, \\
& V_{3}=\frac{1}{2} \alpha_{1}^{2}\left(x_{1}-\beta\right)^{2}+\frac{1}{2} \alpha_{2}^{2}\left(x_{2}-\beta\right)^{2}, \\
& V_{4}=\frac{1}{2} \alpha_{2}^{2}\left(x_{1}+\beta\right)^{2}+\frac{1}{2} \alpha_{1}^{2}\left(x_{2}-\beta\right)^{2}, \\
& V=\frac{V_{1} V_{2} V_{3} V_{4}}{V_{1} V_{2} V_{3}+V_{1} V_{2} V_{4}+V_{1} V_{3} V_{4}+V_{2} V_{3} V_{4}},
\end{aligned}
$$

where $x_{i}$ are not mass scaled. The potential parameters in Eq. (44) are taken as $\beta=2, \alpha_{1}=1.265, \alpha_{2}=2$, and $m=27$. The potential has four minima and possesses a $C_{4}$ symmetry axis, as shown in Fig. 2. Instanton paths connect the neighboring minima as indicated in the figure. The "diagonal" instanton paths have large actions and are negligible. Energy levels split due to tunneling into a triplet, in which the middle level is doubly degenerate. The tunneling splitting pattern consists of energy levels $E_{1}=E_{0}-\Delta, E_{2}=E_{3}=E_{0}$, and $E_{4}=E_{0}+\Delta$, where $\Delta$ corresponds to the tunneling splitting between the neighboring minima and $E_{0}$ is the harmonic energy. We now label the minimum at $(-\beta,-\beta)$ as "left" and the minimum at $(\beta,-\beta)$ as "right." Each instanton path is almost a straight line between two minima; however, because of the anharmonicity, the path is slightly deflected near minima. As a result of this deflection, it enters the left minimum along the lower mode, instead of the higher one, as explained in Appendix B. However, it also possesses a large displacement $\eta$ in Eq. (40) along the higher mode. The higher mode is therefore longitudinal at the left minimum, while the lower mode is longitudinal near the right minimum. As a result, when either of the modes is excited, it cannot be described as a longitudinal or a transversal excitation with respect to the instanton path. It represents the case of longitudinal-transversal excitation, where the excited mode is longitudinal at one minimum and transversal to the path at the other minimum. This case cannot be treated with the method of

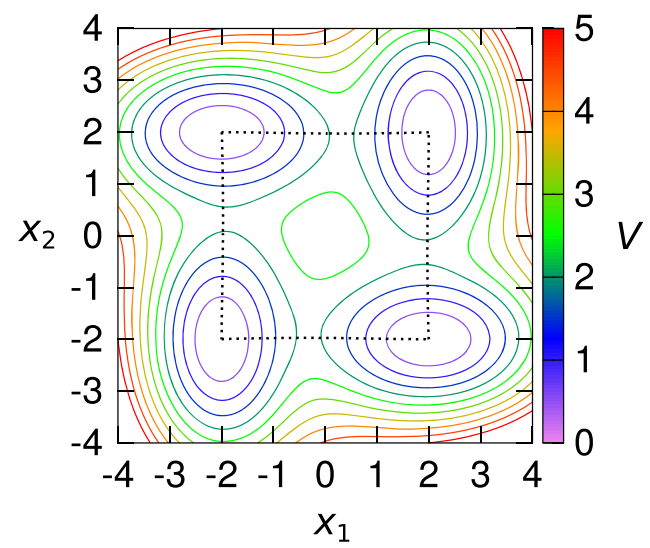

FIG. 2. Potential energy surface for the model potential in Eq. (44) $\left(\alpha_{1}=1.265, \alpha_{2}\right.$ $=2, \beta=2$ ). Superposed on the potential energy surface are the instanton pathways that are responsible for the formation of the tunneling splitting pattern. 
TABLE IV. Tunneling splittings in first two vibrationally excited states $\left(\Delta_{1}\right)$ for the potential in Eq. (44) obtained using instanton theory. Displacements, $\eta$ in Eq. (40), are given for the left $(-\beta,-\beta)$ and the right $(\beta,-\beta)$ minimum. QM labels the exact quantum-mechanical results. The ground-state splitting is $\Delta_{0}=9.129(-12)$, using the JFI method. The exact result is $\Delta_{0}=8.887(-12)$.

\begin{tabular}{lll}
\hline \hline & $\Delta_{1}(1,0)$ & $\Delta_{1}(0,1)$ \\
\hline Instanton & $1.304(-11)$ & $2.979(-11)$ \\
& $1.340(-11)$ & $3.058(-11)$ \\
QM & $1.387(-11)$ & $3.261(-11)$ \\
\hline$\eta^{(\mathrm{L})}$ & $1.775(-11)$ & $6.531(-11)$ \\
$\eta^{(\mathrm{R})}$ & 0.13442 & 0.99092 \\
\hline \hline
\end{tabular}

Ref. 53. The localized wavefunction that corresponds to the longitudinal excitation is of the form $p_{0} \exp \left(-1 / 2 \Delta \mathbf{x}^{\top} \mathbf{A} \Delta \mathbf{x}\right)$, which means that it is even in the dividing plane. On the other hand, the wavefunction that corresponds to the transversal excitation is of the form $\left(\mathbf{U}^{\top} \Delta \mathbf{x}\right) \exp \left(-1 / 2 \Delta \mathbf{x}^{\top} \mathbf{A} \Delta \mathbf{x}\right)$, which is odd in the dividing plane. As a result, the surface integral in the Herring formula is odd and identically equal to zero. It is clear, however, from quantum-mechanical computations that the splitting is not zero but can, in fact, even be larger than the splitting in the ground state, as given in Table IV.

In our treatment, the addition of the $F$ term breaks the symmetry of the wavefunction in the dividing plane, and it moves the node away from the instanton trajectory, while the maximum of the Gaussian part in Eq. (31) stays on the trajectory, as shown in Fig. 3. As a result, the integral in the Herring formula does not vanish. The results obtained using our approach are given in Table IV. From the $\eta$ values in the left minimum, it is clear that, in its vicinity, the instanton trajectory rapidly turns toward the direction of the second (higher) normal mode, while it has to enter the minimum along the first (lower) mode. As a result of this sharp turn, the $F$ value for the left minimum is not zero and, in the end, gives rise to the non-zero tunneling splitting. Contribution of the $\mathbf{Z}$ term in both excited states is quite large compared to its contribution in the

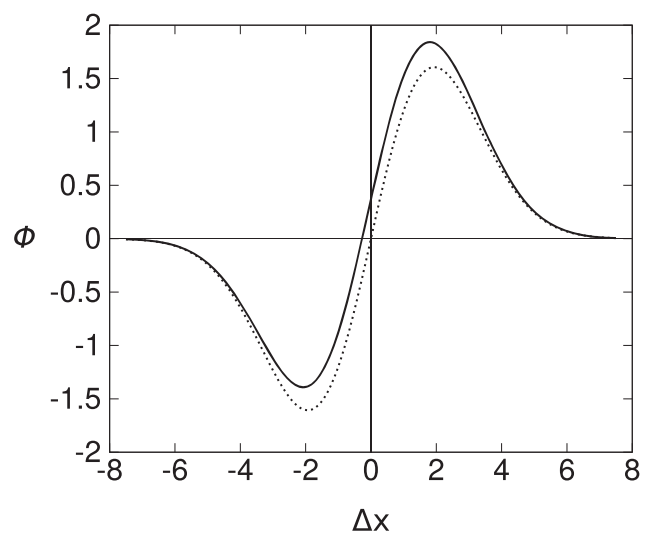

FIG. 3. Comparison of the wavefunctions in the dividing plane (line) obtained by using $U_{\perp}$ only (dotted line) and by using $F+U_{i} \Delta x_{i}$ (full line) in the preexponential factor of the localized wavefunction in Eq. (31). symmetric test case above. This is indicative of the presence of nonnegligible anharmonic effects in this system. The anharmonicity is also a probable reason for the relatively large discrepancies between the instanton and the exact quantum-mechanical results (obtained on the same grid as for the symmetric potential above), where the latter are $28 \%$ and $100 \%$ higher for the excitation of the first and second vibrational modes, respectively. A larger discrepancy in the higher mode could be attributed to its larger energy and the larger spread of its wavefunction into the regions away from the instanton path where anharmonicity is significant.

\section{Water dimer}

The tunneling splitting pattern of water dimer has been extensively studied both experimentally and theoretically, $2,14,25,26$ which makes it a good benchmark system to test our method. We chose the fully deuterated dimer over the non-deuterated one because its vibrational energies are lower. As a consequence, there are more vibrational excitations that do not exceed the barrier height and can be treated with the instanton method. The analytical potential energy surface MB-pol ${ }^{13,75,76}$ was used in all calculations.

Water dimer, shown labeled in Fig. 4, has 8 equivalent symmetry-related and accessible minima, which correspond to the permutations of hydrogen and oxygen atoms that do not break the covalent $\mathrm{H}-\mathrm{O}$ bonds. Permutations that do break the covalent bonds are considered unfeasible. These minima are connected by tunneling rearrangement pathways, ${ }^{77}$ five of which are believed to be responsible for the dimer splitting pattern. ${ }^{2,14,78}$ The acceptor tunneling (AT) path corresponds to permutation (34). In the ground state, its effective barrier is relatively low, $V_{\text {eff }}=77 \mathrm{~cm}^{-1}$, so it gives rise to the largest tunneling matrix element. This matrix element is responsible for the splitting of energy levels into two groups, whose energy difference is called the acceptor splitting $\Delta(\mathrm{A})=4|h(\mathrm{AT})|$. As seen in Table $\mathrm{V}$, the displacements $\eta$ for the AT path lie predominantly along the lowest mode at both minima. The next contribution to the splitting pattern arises from the donor-acceptor interchange via $C_{i}$ and $C_{2}$ symmetry transition states or, alternatively, the geared (GI) and anti-geared (AI) interchange pathways, which correspond to the $(\mathrm{AB})(1324)$ and $(\mathrm{AB})(14)(23)$ permutations, respectively. These pathways have larger effective barriers in the ground state, $V_{\text {eff }}=188 \mathrm{~cm}^{-1}$ and $V_{\text {eff }}=227 \mathrm{~cm}^{-1}$, respectively. They cause the

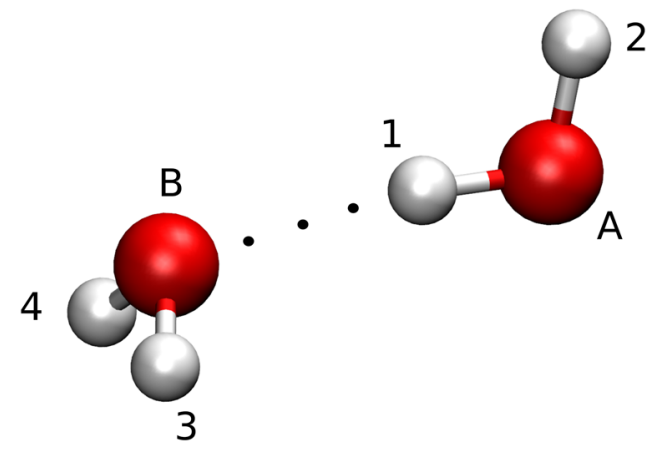

FIG. 4. The minimum energy geometry of the water dimer labeled to represent the reference version. 
TABLE V. Left and right displacements $\eta$ in Eq. (40) in deuterated water dimer for five instanton pathways and excitations into lowest five vibrational modes. Pathways are acceptor tunneling (AT), geared interchange $(\mathrm{Gl})$, antigeared interchange (Al), bifurcation tunneling (BT), and donor exchange (DE).

\begin{tabular}{lccccc}
\hline \hline Mode & AT & GI & AI & BT & DE \\
\hline 1 & 0.99408 & 0.73315 & 0.97661 & 0.00363 & 0.06834 \\
& 0.99413 & 0.73091 & 0.97689 & 0.00205 & 0.97470 \\
\hline 2 & 0.08292 & 0.63492 & 0.14969 & 0.00263 & 0.70957 \\
& 0.08307 & 0.63780 & 0.14916 & 0.00070 & 0.22343 \\
\hline 3 & 0.07001 & 0.24363 & 0.15434 & 0.99927 & 0.70112 \\
& 0.06920 & 0.24284 & 0.15302 & 0.99927 & 0.00373 \\
\hline 4 & 0.00441 & 0.00276 & 0.00135 & 0.03795 & 0.01621 \\
& 0.00449 & 0.00269 & 0.00127 & 0.03792 & 0.00362 \\
\hline 5 & 0.00143 & 0.00163 & 0.00314 & 0.00135 & 0.00032 \\
& 0.00145 & 0.00168 & 0.00307 & 0.00122 & 0.00276 \\
\hline \hline
\end{tabular}

energy levels in both groups, formed by acceptor tunneling, to split into triplets, with the energy width of the lower group called the lower interchange $\Delta(\mathrm{LI})=4|h(\mathrm{GI})+h(\mathrm{AI})|$, while the upper group energy width is called the upper interchange $\Delta(\mathrm{UI})=4 \mid h(\mathrm{GI})-$ $h(\mathrm{AI}) \mid$. The AI path is mostly displaced along the lowest mode near minima as well but has larger projections onto the second and third modes. In contrast, the GI path is almost equally displaced along the first and second modes near minima, while it has to enter the minima along the lowest mode. Finally, the smallest contribution to the splitting pattern of water dimer arises from the bifurcation tunneling (BT) and donor exchange (DE) paths, which correspond to the (12)(34) and (12) permutations, respectively. These pathways possess the highest effective barriers, $V_{\text {eff }}=469 \mathrm{~cm}^{-1}$ and $V_{\text {eff }}=581 \mathrm{~cm}^{-1}$, respectively. They cause the shifts in the energies of the triplets by the amounts called the lower bifurcation and the upper bifurcation, $\Delta(\mathrm{LB})=|h(\mathrm{BT})+4 h(\mathrm{DE})|$ and $\Delta(\mathrm{UB})=\mid h(\mathrm{BT})$ $-4 h(\mathrm{DE}) \mid$. The bifurcation tunneling path is displaced mostly along the third mode near minima. The donor exchange path, on the other hand, is displaced mostly along the lowest mode near one minimum, while it is displaced mostly along the second and third modes near the other minimum. Therefore, this path represents a realistic case of the asymmetric potential that features longitudinal-transversal excitations that we discussed in Subsection IV A on a 2D model potential. The five contributing pathways in water dimer can be found visualized in Refs. 7 and 77.

The lowest mode of vibration in the deuterated water dimer corresponds to donor torsion and has a frequency of $\omega=84 \mathrm{~cm}^{-1}$. In order to calculate the splitting pattern with the excited donor torsion, we calculate the matrix elements, $h=-\Delta_{1} / 2$, for all five rearrangement paths. The AT matrix element, obtained by the instanton method, is 3 times larger than the experimental value, as seen in Table VI. Since donor torsion is the longitudinal mode of the AT path and its excitation frequency is larger than the effective barrier on the path, this represents a case of over-the-barrier tunneling. The instanton method is known to overestimate the splittings by a factor of 2-3 in such circumstances, ${ }^{33,54}$ as also noted in Subsection IV A. The sign of the acceptor splitting is found to be opposite to that of
TABLE VI. Tunneling matrix elements $-h / \mathrm{cm}^{-1}$ for different tunneling pathways in deuterated water dimer $\left(\mathrm{D}_{2} \mathrm{O}\right)_{2}$ obtained using instanton theory. Pathways described are acceptor tunneling (AT), geared interchange (GI), antigeared interchange (Al), bifurcation tunneling (BT), and donor exchange (DE). The excited-state splittings are, top to bottom, obtained using the expansion of $\exp (-w)$ to $F, F+$ $U_{i} \Delta x_{i}$, and $F+U_{i} \Delta x_{i}+\frac{1}{2} Z_{i j} \Delta x_{i} \Delta x_{j}$ terms, respectively. The splittings given in parentheses are experimental ${ }^{79}$ (top) and quantum-mechanical ${ }^{25}$ (bottom) results. (Ground-state (GS) experimental results are from Refs. 25 and 80. .)

\begin{tabular}{lccccc}
\hline \hline Mode & AT & GI & AI & BT & DE \\
\hline GS & 0.766 & $9.73(-3)$ & $4.88(-4)$ & $1.83(-4)$ & $3.21(-6)$ \\
1 & -11.8 & $-4.58(-2)$ & $1.86(-2)$ & $1.43(-9)$ & $2.95(-6)$ \\
& -11.1 & $-5.07(-2)$ & $1.83(-2)$ & $-3.96(-5)$ & $1.12(-5)$ \\
& -12.0 & $-5.30(-2)$ & $1.95(-2)$ & $-3.96(-5)$ & $8.26(-6)$ \\
& $(3.953)$ & $(6.643(-2))$ & $(1.561(-2))$ & $(-)$ & $(-)$ \\
& $(3.92)$ & $(6.63(-2))$ & $(1.63(-2))$ & $(-)$ & $(-)$ \\
\hline 2 & -0.502 & -0.256 & $4.01(-3)$ & $-6.17(-9)$ & $-5.16(-5)$ \\
& -0.509 & -0.254 & $4.98(-3)$ & $-2.28(-4)$ & $-4.38(-5)$ \\
& -0.457 & -0.261 & $5.18(-3)$ & $-2.28(-4)$ & $-4.78(-5)$ \\
& $(0.634)$ & $(0.109)$ & $(1.375(-3))$ & $(-)$ & $(-)$ \\
& $(0.758)$ & $(0.140)$ & $(4.25(-2))$ & $(-)$ & $(-)$ \\
\hline 3 & 1.15 & 0.147 & $2.13(-2)$ & $5.47(-3)$ & $3.27(-6)$ \\
& $2.72(-2)$ & 0.141 & $2.13(-2)$ & $5.42(-3)$ & $2.42(-6)$ \\
& 0.469 & 0.143 & $2.21(-2)$ & $5.58(-3)$ & $5.32(-6)$ \\
& $(0.442)$ & $(3.033(-2))$ & $(2.427(-3))$ & $(-)$ & $(-)$ \\
& $(0.45)$ & $(2.88(-2))$ & $(1.25(-3))$ & $(-)$ & $(-)$ \\
\hline 4 & 19.5 & $2.94(-2)$ & $2.64(-2)$ & $2.17(-3)$ & $-2.43(-4)$ \\
& 8.98 & $3.20(-2)$ & $2.58(-2)$ & $2.41(-3)$ & $-3.70(-4)$ \\
& -57.0 & 0.220 & $-3.87(-2)$ & $6.45(-2)$ & $4.05(-4)$ \\
$(-)$ & $(-)$ & $(-)$ & $(-)$ \\
& $(-)$ & $(-)$ & $(-)$ & $(-)$ \\
\hline \hline & $(1.23)$ & $(0.173)$ & $(7.75(-2))$ & $(-)$ &
\end{tabular}

the ground state, indicating that the groups of states associated with the lower and upper interchange change places. This observation is in agreement with the experimental measurements ${ }^{79}$ and the exact quantum-mechanical calculations.

GI and AI matrix elements are found to be in good agreement with the experimental results ${ }^{79}$ in their absolute values, but their relative sign appears to be wrong. This results in the wrong ordering of the LI and UI splittings in magnitude, as seen in Table VII. We note that the contribution of the $F$ term accounts for $86 \%$ and $95 \%$ of the matrix element in Eq. (33). A large contribution for the AI path is expected as donor torsion is its longitudinal mode. However, for the GI path, which lies along a combination of modes near minima, the contribution of the $F$ term is also important. We presume that the disagreement between the instanton and quantum-mechanical results of Ref. 25 is caused by a large rotation-vibration coupling in the excited mode, which mixes the vibrational states of $K_{a}=0$ and $K_{a}=1$ and is not accounted for in the instanton method. The values obtained for LI and UI $\left(0.134 \mathrm{~cm}^{-1}\right.$ and $\left.0.290 \mathrm{~cm}^{-1}\right)$ are, in fact, in better agreement with the experimental values ${ }^{79}$ for $K_{a}=1$, which are $0.132 \mathrm{~cm}^{-1}$ and $0.257 \mathrm{~cm}^{-1}$, both in magnitude and in ordering.

Lower and upper bifurcations are underestimated for the first excited vibrational mode, as can be seen in Table VII. For the DE path, this represents a longitudinal-transversal excitation, and it 
TABLE VII. Acceptor, upper interchange, and lower interchange splittings $\left(\mathrm{cm}^{-1}\right)$ in deuterated water dimer $\left(\mathrm{D}_{2} \mathrm{O}\right)_{2}$ obtained using the instanton method. The excitedstate splittings are, top to bottom, obtained using the expansion of $\exp (-w)$ to $F$, $F+U_{i} \Delta x_{i}$, and $F+U_{i} \Delta x_{i}+\frac{1}{2} Z_{i j} \Delta x_{i} \Delta x_{j}$ terms, respectively. The splittings given in parentheses are experimental ${ }^{79}$ (top) and quantum-mechanical ${ }^{25}$ (bottom) results. (Ground-state (GS) experimental results are from Refs. 25 and 80.)

\begin{tabular}{|c|c|c|c|c|c|}
\hline Mode & A & UI & LI & UB & LB \\
\hline GS & $\begin{array}{c}3.06 \\
(1.77) \\
(1.78)\end{array}$ & $\begin{array}{l}3.70(-2) \\
(3.6(-2)) \\
(3.6(-2))\end{array}$ & $\begin{array}{l}4.09(-2) \\
(3.9(-2)) \\
(3.8(-2))\end{array}$ & $\begin{array}{c}1.70(-4) \\
(2.2(-4)) \\
\quad(-)\end{array}$ & $\begin{array}{c}1.96(-4) \\
(2.3(-4)) \\
(-)\end{array}$ \\
\hline 1 & $\begin{array}{c}47.3 \\
44.5 \\
47.9 \\
(15.811) \\
(15.68)\end{array}$ & $\begin{array}{c}0.257 \\
0.276 \\
0.290 \\
(0.203) \\
(0.20)\end{array}$ & $\begin{array}{c}0.109 \\
0.129 \\
0.134 \\
(0.328) \\
(0.33)\end{array}$ & $\begin{array}{c}1.18(-5) \\
8.44(-5) \\
7.27(-5) \\
(8.006(-4)) \\
(-)\end{array}$ & $\begin{array}{c}1.18(-5) \\
5.15(-6) \\
6.57(-6) \\
(1.698(-3)) \\
(-)\end{array}$ \\
\hline 2 & $\begin{array}{c}2.01 \\
2.04 \\
1.83 \\
(2.535) \\
(3.03)\end{array}$ & $\begin{array}{c}1.04 \\
1.04 \\
1.07 \\
(0.443) \\
(0.73)\end{array}$ & $\begin{array}{c}1.01 \\
1.00 \\
1.02 \\
(0.432) \\
(0.39)\end{array}$ & $\begin{array}{c}2.06(-4) \\
5.31(-5) \\
3.67(-5) \\
(2.662(-3)) \\
(-)\end{array}$ & $\begin{array}{c}2.06(-4) \\
4.03(-4) \\
4.20(-4) \\
2.635(-3) \\
\quad(-)\end{array}$ \\
\hline 3 & $\begin{array}{c}4.60 \\
0.109 \\
1.88 \\
(1.768) \\
(1.81)\end{array}$ & $\begin{array}{c}0.503 \\
0.479 \\
0.484 \\
(0.112) \\
(0.11)\end{array}$ & $\begin{array}{c}0.673 \\
0.649 \\
0.660 \\
(0.131) \\
(0.12)\end{array}$ & $\begin{array}{c}5.46(-3) \\
5.41(-3) \\
5.56(-3) \\
(1.304(-3)) \\
(-)\end{array}$ & $\begin{array}{c}5.49(-3) \\
5.43(-3) \\
5.60(-3) \\
5.174(-3) \\
\quad(-)\end{array}$ \\
\hline 4 & $\begin{array}{l}78.1 \\
35.9 \\
228 \\
(-) \\
(4.9)\end{array}$ & $\begin{array}{c}0.012 \\
0.025 \\
1.04 \\
(-) \\
(0.38)\end{array}$ & $\begin{array}{c}0.22 \\
0.231 \\
0.725 \\
(-) \\
(1.0)\end{array}$ & $\begin{array}{c}3.14(-3) \\
3.89(-3) \\
6.29(-2) \\
(-) \\
(-)\end{array}$ & $\begin{array}{c}1.20(-3) \\
9.35(-4) \\
6.62(-2) \\
(-) \\
(-)\end{array}$ \\
\hline
\end{tabular}

was shown for the model potential above that an underestimate is expected because of the unaccounted anharmonicities. However, the difference between the lower and upper bifurcation is not zero, as it would be in using the theory of Ref. 53, and even though it is underestimated, a rough estimate of its value is obtained. The exact quantum-mechanical calculations ${ }^{25}$ do not report it, probably due to the difficulty in converging the values with sufficient accuracy. It is also worth mentioning that the $\mathrm{UB}$ and $\mathrm{LB}$ change significantly in the $K_{a}=1$ rotational state to $8.906(-4) \mathrm{cm}^{-1}$ for UB and $1.201(-4) \mathrm{cm}^{-1}$ for LB. These values are again in better agreement with those that we computed, as in the case of the AT path, which provides further indication that the coupling of the first excited state to rotations plays a significant role. Finally, the UB and LB are underestimated even in the ground vibrational state, which suggests the possibility that the BT and DE pathways are poorly described by the PES, either by too large potential energy barriers or by slightly misplaced instanton paths, both of which can have a drastic effect on the splittings.

The second mode corresponds to the acceptor twist, with frequency $\omega=100 \mathrm{~cm}^{-1}$, while the third mode corresponds to the acceptor wag, with frequency $\omega=110 \mathrm{~cm}^{-1}$. However, in quantummechanical calculations, ${ }^{25}$ the order of these two motions changes, and the acceptor wag frequency drops to $82 \mathrm{~cm}^{-1}$, while the acceptor twist drops to $90 \mathrm{~cm}^{-1}$. The large deviation of vibrational energies from the harmonic frequencies is a strong indication of large anharmonic effects in these two vibrational modes. Furthermore, since their energy difference is very small, it was noticed that these states interact through a Coriolis perturbation ${ }^{79}$ adding to the quantitative disagreement with the harmonic analysis. Nevertheless, the splittings obtained from the second excited mode are in good agreement with the experimental results. We note that the $F$ term on the AI path contributes with around $77 \%$ to the matrix element, even though the displacements near minima along this mode are small. The overestimation of the GI matrix element can be explained by the fact that the path has a large projection onto the second mode near minima, which means that the effective barrier is significantly lowered. The discrepancy of the AI matrix element can be explained by the inaccuracy of the PES, since quantum-mechanical results ${ }^{25}$ on a similar surface ${ }^{81}$ also overestimate this matrix element. Upper and lower bifurcations are again underestimated, probably for the same reasons as above, namely, the inadequate PES and the unaccounted anharmonic effects in the longitudinal-transversal excitation.

In the case of the third mode excitation, especially interesting is the AT path for which the contributions of the $F$ term and the $\mathbf{U}$ term in the matrix element almost cancel each other out, while the major contribution arises from the anharmonicity contained in the $\mathbf{Z}$ term. For this excitation, both GI and AI matrix elements are overestimated. This can again be attributed to the rovibrational coupling since the quantum-mechanical results show a significant increase in the lower and upper interchange with the excitation to the $K_{a}=1$ rotational state. ${ }^{25}$ Upper and lower bifurcations for this excitation show a much better agreement with the experimental values ${ }^{79}$ than above.

At larger excitation frequencies, the theory breaks down. A probable cause of this breakdown is the fact that as the frequency increases, the contribution of the $w$ term to the overall splitting rises significantly. This is due to the fact that the $F$ contribution depends exponentially on the frequency of excitation, while the $\eta$ values do not compensate it. As a result, its contribution becomes comparable to that of $W_{0}$, while the WKB approach assumes $\ln F \ll W_{0}$. A good test of the reliability of the obtained results is to redo the calculations with a different value of the initial "jump" parameter $\varepsilon$. As the value of $\varepsilon$ is reduced, the results should converge to the correct value. However, there is a limit to how much $\varepsilon$ can be reduced, as the propagation from the point too close to the minimum is not stable. ${ }^{52,}$ If the results converge before this breakdown, they can be treated as reliable. As the value of $\varepsilon$ is increased, the values of the splittings should not change by more than a few percent. This is the case for the excitations in the first three lowest modes. For the fourth excited mode, if we change $\varepsilon$ from $0.1 \mathrm{~m}^{1 / 2} a_{0}$ to $1 \mathrm{~m}^{1 / 2} a_{0}$, the AT matrix element changes from $8.98 \mathrm{~cm}^{-1}$ to $3(+3) \mathrm{cm}^{-1}$, which is an indication that the breakdown of theory occurred. Similar behavior is present for the AI pathway, where the matrix element changes from $2.58(-2) \mathrm{cm}^{-1}$ to $0.23 \mathrm{~cm}^{-1}$. The change is not as drastic as in the AT case, but it indicates that the error bars on our results are very large, which also explains the discrepancies of results for the LI and UI splittings. Noticeable changes are also present for the DE pathway [from $-3.70(-4) \mathrm{cm}^{-1}$ to $-6.85(-4) \mathrm{cm}^{-1}$ ], while the values for other pathways do not change appreciably and can be considered reliable. 


\section{CONCLUSIONS}

We developed a semiclassical theory for calculating tunneling splittings of low-lying vibrationally excited states based on the instanton method. A WKB wavefunction is constructed along the instanton path and its harmonic neighborhood for each well and inserted into the Herring formula to obtain the splitting that matches the JFI result in the ground state. ${ }^{67}$ The excited-state splittings are then obtained constructing excited-state wavefunctions analogously. The procedure closely follows that of Ref. 53 but uses a more general boundary condition near minima and does not assume the leftright mirror symmetry of potential along the instanton path. In our approach, transversal and longitudinal excitations do not require separate treatments, as in Ref. 53. This allows us to compute splittings in the systems where the excited vibrational mode does not line up along the instanton path near minima, but has both longitudinal and transversal components, or the systems in which the excited mode is longitudinal at one minimum and transversal at the other. Both components are propagated simultaneously along the instanton path, and cross interaction is kept in the treatment.

The tests on the symmetric double-well model potential showed that a high accuracy can be expected for low-lying states below the barrier. It was shown that for transversal modes, even a small longitudinal displacement near minima can dominate the tunneling splitting. We also observed that the longitudinaltransversal cross terms improve results. The tests on the asymmetric model potential showed that we can calculate splitting estimates for excited longitudinal-transversal modes, albeit with somewhat reduced accuracy. Finally, we calculated the tunneling splitting pattern of the deuterated water dimer in vibrationally excited lowest three modes by computing contributions from five different rearrangement pathways. This is a particularly challenging system for treatment with partly harmonic theories. Additionally, the system exhibits significant rovibrational couplings, which are, at present, neglected in our treatment. We could nevertheless obtain reasonable agreement in many cases in a system that showcases the situations in which the present theory gives significantly different results from that of Ref. 53.

Tunneling splittings in vibrationally excited states require no additional information about the molecular system. All computational effort is concentrated, as for the ground-state splittings, in determining the MAP by optimization and the evaluation of Hessians along the MAP. This allows us to compute and interpret splitting patterns in many mid-sized molecules using state-ofthe-art potentials. The theory is applied in Cartesian coordinates and requires no modification for treating different molecular systems. However, tunneling splittings in vibrational states with higher frequencies, such as the excitations of librational modes of water trimer $^{15}$ and pentamer ${ }^{16}$ that were recently measured, cannot be treated with the theory in the present format. Many small tunneling systems exhibit large rotation-vibration coupling, which is currently neglected and can affect the splittings. A computationally tractable theory for calculating splittings in rotationally excited states would also be desirable. These are some of the immediate challenges remaining in which the future efforts will certainly be directed in a quest to provide quantitative estimates for splitting patterns for molecules and clusters that are out of reach to the exact quantum-mechanical treatments.

\section{ACKNOWLEDGMENTS}

This work was supported by the Croatian Science Foundation under Grant No. IP-2016-06-1142 and, in part, by the QuantiXLie Centre of Excellence, a project cofinanced by the Croatian Government and European Union through the European Regional Development Fund-The Competitiveness and Cohesion Operational Programme (Grant No. KK.01.1.1.01.0004).

\section{APPENDIX A: METHOD OF CHARACTERISTICS AND LOCAL COORDINATES}

The method of characteristics is a technique for solving partial differential equations. ${ }^{82}$ It relies on locating curves, the characteristics, along which the gradient of the desired solution is tangential. As a consequence, the partial differential equation reduces to an ordinary differential equation. For a non-linear partial differential equation of the form

$$
F\left(x_{1}, \ldots, x_{N}, p_{1}, \ldots, p_{N}, f\right)=0,
$$

where $p_{i}=\partial f / \partial x_{i}$, defining equations of the characteristics are

$$
\begin{aligned}
\frac{\mathrm{d} x_{i}}{\mathrm{~d} \tau} & =\frac{\partial F}{\partial p_{i}}, \\
\frac{\mathrm{d} p_{i}}{\mathrm{~d} \tau} & =-\frac{\partial F}{\partial x_{i}}-\frac{\partial F}{\partial f} p_{i}, \\
\frac{\mathrm{d} f}{\mathrm{~d} \tau} & =\frac{\partial F}{\partial p_{i}} p_{i},
\end{aligned}
$$

where $\tau$ parametrizes the characteristic.

The Hamilton-Jacobi equation is a non-linear partial differential equation for which $F=\frac{1}{2} p_{i} p_{i}-V$, where $p_{i}=\partial W_{0} / \partial x_{i}$. Its characteristics are therefore

$$
\begin{aligned}
& \frac{\mathrm{d} x_{i}}{\mathrm{~d} \tau}=p_{i}, \\
& \frac{\mathrm{d} p_{i}}{\mathrm{~d} \tau}=\frac{\partial V}{\partial x_{i}} .
\end{aligned}
$$

The characteristics describe classical trajectories on the inverted PES, while $\nabla W_{0}$ is the momentum on the trajectory. The total energy of the classical motion is $E_{\text {tot }}=\frac{1}{2} p_{i} p_{i}+(-V)=0$. On characteristics, $W_{0}$ is found by solving

$$
\frac{\mathrm{d} W_{0}}{\mathrm{~d} \tau}=p_{i} p_{i}=2 \mathrm{~V}
$$

The parameter $\tau$ represents time, and as the trajectory approaches minimum, its value $\tau \rightarrow-\infty$. This is numerically problematic, so we reparametrize characteristics with the arc length distance from the minimum, $S$, using the transformation in Eq. (7).

In order to expand $W_{0}$ in Taylor series around the characteristic, it is convenient to define a set of local coordinates $\{S, \Delta \mathbf{x}\}$. Since coordinate $S$ parametrizes characteristic, it is only defined for the points lying on it. In order to assign a value $S$ to the point that does not lie on the characteristic, a point $\mathbf{x}_{0}(S)$ that does lie on it is chosen so that

$$
\left(x_{i}-x_{0 i}(S)\right) p_{0 i}=0,
$$

that is, $\mathbf{x}_{0}(S)$ is chosen so that the vector connecting it with the point $\mathbf{x}$ is orthogonal to the characteristic at $\mathbf{x}_{0}(S)$. The value of $S$ that 
corresponds to $\mathbf{x}_{0}(S)$ is then assigned to $\mathbf{x}$. The orthogonal coordinates $\Delta \mathbf{x}$ are then defined as $\Delta \mathbf{x}=\mathbf{x}-\mathbf{x}_{0}(S)$. Differentiation of Eq. (A5) gives $^{16}$

$$
\frac{\partial S}{\partial x_{i}}=\frac{\frac{p_{0 i}}{p_{0}}}{1-\frac{\mathbf{a}^{\top} \Delta \mathbf{x}}{p_{0}^{2}}}=\frac{p_{0 i}}{p_{0}}\left(1+\frac{\mathbf{a}^{\top} \Delta \mathbf{x}}{p_{0}^{2}}+\ldots\right),
$$

where $\mathbf{a}=\frac{\mathrm{d} \mathbf{p}_{0}}{\mathrm{~d} \tau}$ denotes the acceleration. From the differentiation of the Hamilton-Jacobi equation [Eq. (3)], we obtain $\mathbf{a}=\mathbf{A} \mathbf{p}_{0}$. Finally, the differentiation of the defining equation of orthogonal coordinates in Eq. (A5) gives the transformation

$$
\frac{\partial \Delta x_{i}}{\partial x_{j}}=\delta_{i j}-\frac{p_{0 i} p_{0 j}}{p_{0}^{2}}\left(1+\frac{\mathbf{a}^{\top} \Delta \mathbf{x}}{p_{0}^{2}}+\ldots\right) .
$$

Equations (A6) and (A7) are used throughout the paper to transform between Cartesian and local coordinates on the characteristic as $\mathbf{A}$, $\mathbf{U}, \mathbf{B}$, and $\mathbf{Z}$ are all given in the differential form.

\section{APPENDIX B: WAVEFUNCTIONS NEAR MINIMA}

Near minima $\mathbf{x}_{\min }$, the PES can be approximated by a harmonic oscillator potential

$$
V=\frac{1}{2} \omega_{i}^{2} q_{i}^{2}
$$

where $q_{i}=V_{j i}\left(x_{j}-x_{\min j}\right)$ are normal coordinates and $\omega_{i}$ are corresponding harmonic frequencies. Since $\mathbf{A}_{0}=\mathbf{H}^{1 / 2}$, we have $\mathbf{V}^{\top} \mathbf{A}_{0} \mathbf{V}$ $=\boldsymbol{\Omega}$, with $(\boldsymbol{\Omega})_{i j}=\omega_{i} \delta_{i j}$. In the harmonic region near minima, the equations of characteristics, Eq. (A3) become

$$
\begin{aligned}
& \frac{\mathrm{d}^{2} q_{i}}{\mathrm{~d} \tau^{2}}=\frac{\partial V}{\partial q_{i}}, \\
& \frac{\mathrm{d}^{2} q_{i}}{\mathrm{~d} \tau^{2}}=\omega_{i}^{2} q_{i} .
\end{aligned}
$$

The trajectory along the characteristic from the minimum to an arbitrary point $\mathbf{q}_{1}$ at $\tau=0$ inside the harmonic region is

$$
q_{i}(\tau)=q_{1 i} \mathrm{e}^{\omega_{i} \tau} .
$$

By considering the tangent vector of the characteristic,

$$
t_{i}=\frac{p_{0 i}}{p_{0}}=\frac{\omega_{i} q_{1 i} \mathrm{e}^{\omega_{i} \tau}}{\sqrt{\omega_{j}^{2} q_{1 j}^{2} \mathrm{e}^{2 \omega_{j} \tau}}},
$$

we note that in the limit $\tau \rightarrow-\infty$, the tangent becomes $t_{i}=\delta_{i M}$, where $M$ denotes the lowest frequency normal mode for which $q_{1, M}$ $\neq 0$. This means that all characteristics approach the minimum along the lowest normal mode with a non-zero projection upon entering the harmonic region. In the case of an $n$-fold degeneracy of the mode, the minimum is approached in the $n$-dimensional surface defined by the corresponding eigenvectors, as shown in a similar analysis in Ref. 83.

The function $W_{0}$ in Eq. (A4) can be evaluated in the harmonic region at the characteristic as

$$
W_{0}(\tau)=\int_{-\infty}^{\tau} \omega_{j}^{2} q_{1 j}^{2} \mathrm{e}^{2 \omega_{j} \tau^{\prime}} \mathrm{d} \tau^{\prime}
$$

or, making use of Eq. (B3), as

$$
W_{0}(\mathbf{q})=\frac{1}{2} \omega_{j} q_{j}^{2}
$$

Furthermore, since in the harmonic region $\mathbf{A} \approx \mathbf{A}_{0}$, the ground-state wavefunction corresponds to that of the harmonic oscillator,

$$
\phi=\mathrm{e}^{-\frac{1}{2} \omega_{j} q_{j}^{2}}
$$

Equation (B7) is used to approximate the norm of the ground-state wavefunction in the Herring formula [Eq. (1)].

For vibrationally excited states, the correct form of the wavefunction at the minimum is obtained by choosing $\left(\mathbf{U}_{0}\right)_{i}=V_{i e}$, that is, by equating the vector $\mathbf{U}$ with the excited normal mode at the minimum. The wavefunction then has the form

$$
\phi=q_{\mathrm{e}} \mathrm{e}^{-\frac{1}{2} \omega_{j} q_{j}^{2}} .
$$

For a point on the characteristic, which lies in the harmonic region, $\Delta x_{i}=0$, so its form is

$$
\phi=F(\varepsilon) \mathrm{e}^{\frac{1}{2} \omega_{j} q_{j}^{2}(\varepsilon)} .
$$

Therefore, the initial condition for the $F$ term at $S=\varepsilon$ has to be

$$
F(\varepsilon)=q_{\mathrm{e}}=\mathbf{U}_{0}^{\top}(\mathbf{x}(\varepsilon)-\mathbf{x}(0)),
$$

in order to yield the correct form of the wavefunction in Eq. (B8).

\section{APPENDIX C: ANHARMONICITY ABOUT THE INSTANTON PATH}

The anharmonicity of potential in the directions perpendicular to the instanton path can be partially accounted for by including the higher derivatives of the PES along the instanton path, beyond Hessian, in the semiclassical treatment of Sec. III. We assume below that the third derivative tensor of the PES with elements $c_{i j k}=\frac{\partial^{3} V}{\partial x_{i} \partial x_{j} \partial x_{k}}$ along the instanton path has been determined. This allows us to compute the third derivatives of function $W_{0}, B_{i j k}=\frac{\partial^{3} W_{0}}{\partial x_{i} \partial x_{j} \partial x_{k}}$, in Taylor expansion in Eq. (10). The equation for propagation of tensor $\mathbf{B}$ is obtained by differentiating the Hamilton-Jacobi equation [Eq. (3)] three times as

$$
\begin{gathered}
\frac{\partial^{4} W_{0}}{\partial x_{i} \partial x_{j} \partial x_{k} \partial x_{l}} \frac{\partial W_{0}}{\partial x_{l}}+\frac{\partial^{3} W_{0}}{\partial x_{i} \partial x_{j} \partial x_{l}} \frac{\partial^{2} W_{0}}{\partial x_{l} \partial x_{k}}+\frac{\partial^{3} W_{0}}{\partial x_{i} \partial x_{l} \partial x_{k}} \frac{\partial^{2} W_{0}}{\partial x_{l} \partial x_{j}} \\
+\frac{\partial^{3} W_{0}}{\partial x_{l} \partial x_{j} \partial x_{k}} \frac{\partial^{2} W_{0}}{\partial x_{l} \partial x_{i}}=\frac{\partial^{3} V}{\partial x_{i} \partial x_{j} \partial x_{k}} .
\end{gathered}
$$

The first term in Eq. (C1) represents a directional derivative of the tensor element $B_{i j k}$ along the instanton trajectory, while the other terms can be recognized as tensor elements of $\mathbf{B}$ and of Hessian A, which is determined by solving Eq. (9). Equation (C1) on the instanton reads

$$
p_{0} B_{i j k}^{\prime}+B_{i j l} A_{l k}+B_{i l k} A_{l j}+B_{l j k} A_{l i}=c_{i j k} .
$$

We proceed to determine the initial condition $\mathbf{B}(\varepsilon)$ in the vicinity of the minimum. For that purpose, we linearize Eq. (C2), 
following an analogous procedure to that for $\mathbf{A}$ in Refs. 52 and 67, as

$$
\begin{aligned}
\mathbf{B} & =\mathbf{B}^{(0)}+\mathbf{B}^{(1)} S, \\
\mathbf{c} & =\mathbf{c}^{(0)}+\mathbf{c}^{(1)} S, \\
\mathbf{A} & =\mathbf{A}^{(0)}+\mathbf{A}^{(1)} S, \\
p_{0} & =p_{0}^{(1)} S .
\end{aligned}
$$

Inserting the above expressions into Eq. (C2) and equating terms of the same order in $S$ yields equations for $\mathbf{B}^{(0)}$ and $\mathbf{B}^{(1)}$ as

$$
\begin{aligned}
B_{i j l}^{(0)} A_{l k}^{(0)}+B_{i l k}^{(0)} A_{l j}^{(0)}+B_{l j k}^{(0)} A_{l i}^{(0)}= & c_{i j k}^{(0)}, \\
p_{0}^{(1)} B_{i j k}^{(1)}+B_{i j l}^{(1)} A_{l k}^{(0)}+B_{i l k}^{(1)} A_{l j}^{(0)}+B_{l j k}^{(1)} A_{l i}^{(0)}= & c_{i j k}^{(1)}-B_{i j l}^{(0)} A_{l k}^{(1)} \\
& -B_{i l k}^{(0)} A_{l j}^{(1)}-B_{l j k}^{(0)} A_{l i}^{(1)} .
\end{aligned}
$$

These are solved by transforming to the basis of normal modes, the eigenvectors of $\mathbf{A}^{(0)}$, using the following relations:

$$
\begin{aligned}
& \omega_{i} \delta_{i j}=V_{i^{\prime} i} V_{j^{\prime} j} A_{i^{\prime} j^{\prime}}^{(0)}, \\
& \tilde{B}_{i j k}^{(0)}=V_{i^{\prime} i} V_{j^{\prime} j} V_{k^{\prime} k} B_{i^{\prime} j^{\prime} k^{\prime}}^{(0)}, \\
& \tilde{c}_{i j k}^{(0)}=V_{i^{\prime} i} V_{j^{\prime} j} V_{k^{\prime} k} c_{i^{\prime} j^{\prime} k^{\prime}}^{(0)} .
\end{aligned}
$$

Inserting Eq. (C5) into Eq. (C4) yields equations

$$
\begin{aligned}
& \tilde{B}_{i j k}^{(0)}=\frac{\tilde{c}_{i j k}^{(0)}}{\omega_{i}+\omega_{j}+\omega_{k}}, \\
& \tilde{B}_{i j k}^{(1)}=\frac{\tilde{c}_{i j k}^{(1)}-\tilde{B}_{i j l}^{(0)} \tilde{A}_{l k}^{(1)}-\tilde{B}_{i l k}^{(0)} \tilde{A}_{l j}^{(1)}-\tilde{B}_{l j k}^{(0)} \tilde{A}_{l i}^{(1)}}{p_{0}^{(1)}+\omega_{i}+\omega_{j}+\omega_{k}},
\end{aligned}
$$

which are needed to construct $\mathbf{B}(\varepsilon)$. Equation $(\mathrm{C} 2)$ can now be solved in the interval $[\varepsilon, S]$ using any differential equation solver, such as the Runge-Kutta method.

Tensor B cannot be included in the wavefunction of Eq. (2) without the inclusion of fourth derivatives as the resulting wavefunction would not be integrable in the dividing plane. However, it is used below to compute the $\mathbf{Z}$ term in the expansion of $\exp (-w)$ in Eq. (34) and thus indirectly account for a part of anharmonicity.

We first note that the following expressions are valid on the instanton path:

$$
\begin{aligned}
F & =\mathrm{e}^{-w}, \\
U_{i} & =\frac{\partial}{\partial x_{i}} \mathrm{e}^{-w}=-\frac{\partial w}{\partial x_{i}} \mathrm{e}^{-w}, \\
Z_{i j} & =\frac{\partial^{2}}{\partial x_{i} \partial x_{j}} \mathrm{e}^{-w}=-\frac{\partial^{2} w}{\partial x_{i} \partial x_{j}} \mathrm{e}^{-w}+\frac{\partial w}{\partial x_{i}} \frac{\partial w}{\partial x_{j}} \mathrm{e}^{-w}, \\
Z_{i j} p_{j} & =\left(\frac{\partial}{\partial x_{j}} U_{i}\right) p_{j}=p_{0} U_{i}^{\prime} .
\end{aligned}
$$

In the next step, we differentiate Eq. (19) twice to obtain useful relations,

$$
\begin{aligned}
p_{k} \frac{\partial^{2} w}{\partial x_{i} \partial x_{k}} \mathrm{e}^{-w}= & A_{i k} U_{k}, \\
p_{k} \frac{\partial^{3} w}{\partial x_{i} \partial x_{j} \partial x_{k}} \mathrm{e}^{-w}= & B_{i j k} U_{k}+A_{i k} Z_{k j}+A_{j k} Z_{k i} \\
& +\frac{\partial w}{\partial x_{j}} A_{i k} U_{k}+\frac{\partial w}{\partial x_{i}} A_{j k} U_{k} .
\end{aligned}
$$

Finally, we take the third derivative of $\exp (-w)$ in Eq. (34) to arrive at

$$
\begin{aligned}
\left(\frac{\partial}{\partial x_{k}} Z_{i j}\right) p_{k}= & -p_{k} \frac{\partial^{3} w}{\partial x_{i} \partial x_{j} \partial x_{k}} \mathrm{e}^{-w}+p_{k} \frac{\partial w}{\partial x_{k}} \frac{\partial^{2} w}{\partial x_{i} \partial x_{j}} \mathrm{e}^{-w} \\
& +p_{k} \frac{\partial w}{\partial x_{i}} \frac{\partial^{2} w}{\partial x_{k} \partial x_{j}} \mathrm{e}^{-w}+p_{k} \frac{\partial w}{\partial x_{j}} \frac{\partial^{2} w}{\partial x_{i} \partial x_{k}} \mathrm{e}^{-w} \\
& -p_{k} \frac{\partial w}{\partial x_{k}} \frac{\partial w}{\partial x_{i}} \frac{\partial w}{\partial x_{j}} \mathrm{e}^{-w}
\end{aligned}
$$

where we insert Eq. (C9) and recognize Eq. (C8) to obtain the equation for $\mathbf{Z}$ in the following form:

$$
p_{0} Z_{i j}^{\prime}+A_{i k} Z_{k j}+A_{j k} Z_{k i}+B_{i j k} U_{k}+\omega_{\mathrm{e}} Z_{i j}=0
$$

This equation is again solved separately in the interval $[0, \varepsilon]$ and $[\varepsilon, S]$, following the same procedure as for $\mathbf{A}$ and $\mathbf{B}$. All objects are expanded up to linear terms in $S$ and inserted into Eq. (C11). By equating terms of the same order in $S$, we obtain equations for $Z^{(0)}$ and $\mathbf{Z}^{(1)}$,

$$
\begin{aligned}
& \mathbf{A}^{(0)} \mathbf{Z}^{(0)}+\mathbf{Z}^{(0)} \mathbf{A}^{(0)}+\omega_{\mathrm{e}} \mathbf{Z}^{(0)}+\mathbf{B}^{(0)} \mathbf{U}^{(0)}=0 \\
& p_{0}^{(1)} \mathbf{Z}^{(1)}+\mathbf{A}^{(0)} \mathbf{Z}^{(1)}+\mathbf{Z}^{(1)} \mathbf{A}^{(0)}+\omega_{\mathrm{e}} \mathbf{Z}^{(1)}+\mathbf{A}^{(1)} \mathbf{Z}^{(0)}+\mathbf{Z}^{(0)} \mathbf{A}^{(1)} \\
& \quad+\mathbf{B}^{(1)} \mathbf{U}^{(0)}+\mathbf{B}^{(0)} \mathbf{U}^{(1)}=0
\end{aligned}
$$

where matrices $(\mathbf{B U})_{i j}$ evaluate as $B_{i j k} U_{k}$. These equations are again solved by transforming to the basis of normal modes, the eigenvectors of $\mathbf{A}^{(0)}$, as

$$
\begin{aligned}
& \tilde{Z}_{i j}^{(0)}=-\frac{\left(\tilde{B}^{(0)} \tilde{U}^{(0)}\right)_{i j}}{\omega_{\mathrm{e}}+\omega_{i}+\omega_{j}}, \\
& \tilde{Z}_{i j}^{(1)}=-\frac{\left(\tilde{B}^{(1)} \tilde{U}^{(0)}\right)_{i j}+\left(\tilde{B}^{(0)} \tilde{U}^{(1)}\right)_{i j}+\left(\tilde{A}^{(1)} \tilde{Z}^{(0)}\right)_{i j}+\left(\tilde{Z}^{(0)} \tilde{A}^{(1)}\right)_{i j}}{p_{0}^{(1)}+\omega_{\mathrm{e}}+\omega_{i}+\omega_{j}} .
\end{aligned}
$$

We are now in the position to compute $\mathbf{Z}(\varepsilon)$, which serves as the initial condition for the propagation of $\mathbf{Z}$ in the interval $[\varepsilon, S]$ by solving Eq. (C11) using the Runge-Kutta method.

When the $\mathbf{Z}$ term is included in the expansion of $\exp (-w)$, the tunneling splitting formula assumes the following form:

$$
\begin{aligned}
\Delta_{1}= & \Delta_{0}\left(2 \omega_{\mathrm{e}}\right)\left(F^{(\mathrm{L})} F^{(\mathrm{R})}+\frac{1}{2} \mathbf{U}^{(\mathrm{L}) \top} \overline{\mathbf{A}}^{-1} \mathbf{U}^{(\mathrm{R})}\right. \\
& \left.+\frac{1}{4} F^{(\mathrm{L})} \operatorname{Tr}\left(\mathbf{Z}^{(\mathrm{R})} \overline{\mathbf{A}}^{-1}\right)+\frac{1}{4} F^{(\mathrm{R})} \operatorname{Tr}\left(\mathbf{Z}^{(\mathrm{L})} \overline{\mathbf{A}}^{-1}\right)\right),
\end{aligned}
$$


where terms of the form $Z_{i j} Z_{k l} \Delta x_{i} \Delta x_{j} \Delta x_{k} \Delta x_{l}$ in the surface integral have been neglected as their contribution was found to be negligible.

\section{APPENDIX D: INVARIANCE OF TUNNELING SPLITTINGS WITH RESPECT TO THE POSITION OF THE DIVIDING PLANE}

The invariance of the ground-state tunneling splitting formula can be proved by differentiating Eq. (17) with respect to the position of the connection point $S_{\mathrm{cp}}$, where the dividing plane intersects the instanton path,

$$
\begin{aligned}
\frac{\partial \Delta_{0}}{\partial S_{\mathrm{cp}}}= & \frac{\Delta_{0}}{2 p_{0}}\left(2 \frac{\partial p_{0}}{\partial S_{\mathrm{cp}}}-\frac{p_{0}}{\operatorname{det}^{\prime} \overline{\mathbf{A}}} \frac{\partial}{\partial S_{\mathrm{cp}}} \operatorname{det}^{\prime} \overline{\mathbf{A}}-2 p_{0} \frac{\partial}{\partial S_{\mathrm{cp}}} W_{1}^{(\mathrm{L})}\right. \\
& \left.-2 p_{0} \frac{\partial}{\partial S_{\mathrm{cp}}} W_{1}^{(\mathrm{R})}\right) .
\end{aligned}
$$

The derivative of the determinant in Eq. (D1) is simplified using the Jacobi formula [Eq. (C4) in Ref. 67], while $W_{1}^{\mathrm{L} / \mathrm{R}}$ functions are differentiated in the upper/lower limit of the integral in Eq. (11),

$$
\begin{aligned}
\frac{\partial \Delta_{0}}{\partial S_{\mathrm{cp}}}= & \frac{\Delta_{0}}{2 p_{0}}\left(2 \frac{\partial p_{0}}{\partial S_{\mathrm{cp}}}-\operatorname{Tr}\left(\overline{\mathbf{A}}^{-1} p_{0} \frac{\partial}{\partial S_{\mathrm{cp}}} \overline{\mathbf{A}}\right)-\operatorname{Tr}\left(\mathbf{A}^{(\mathrm{L})}-\mathbf{A}_{0}\right)\right. \\
& \left.+\operatorname{Tr}\left(\mathbf{A}^{(\mathrm{R})}-\mathbf{A}_{0}\right)\right) .
\end{aligned}
$$

The derivative of $\overline{\mathbf{A}}$ can be shown to equal

$$
p_{0} \frac{\partial}{\partial S_{\mathrm{cp}}} \overline{\mathbf{A}}=\frac{1}{2} \overline{\mathbf{A}}\left(\mathbf{A}^{(\mathrm{R})}-\mathbf{A}^{(\mathrm{L})}\right)+\frac{1}{2}\left(\mathbf{A}^{(\mathrm{R})}-\mathbf{A}^{(\mathrm{L})}\right) \overline{\mathbf{A}},
$$

where use has been made of Eqs. (9) and (16). Furthermore, since the tangent $\mathbf{t}$ is an eigenvector of $\overline{\mathbf{A}}$ with zero eigenvalue and, by definition of the pseudoinverse, $\overline{\mathbf{A}}^{-1} \mathbf{t}=0$, we have $\mathbf{P} \overline{\mathbf{A}} \mathbf{P}=\overline{\mathbf{A}}$ and $\mathbf{P} \overline{\mathbf{A}}^{-1} \mathbf{P}=\overline{\mathbf{A}}^{-1}$, where $\mathbf{P}=\mathbf{I}-\mathbf{t t}^{\top}$ is the operator that projects out the tangent of the instanton path. Using the above, one can show that

$$
\begin{aligned}
\operatorname{Tr}\left(\overline{\mathbf{A}}^{-1} p_{0} \frac{\partial}{\partial S_{\mathrm{cp}}} \overline{\mathbf{A}}\right) & =\operatorname{Tr}\left(\mathbf{P}\left(\mathbf{A}^{(\mathrm{R})}-\mathbf{A}^{(\mathrm{L})}\right) \mathbf{P}\right) \\
& =\operatorname{Tr}\left(\mathbf{A}_{\perp}^{(\mathrm{R})}-\mathbf{A}_{\perp}^{(\mathrm{L})}\right) .
\end{aligned}
$$

Thus, the derivative of the tunneling splitting becomes

$$
\frac{\partial \Delta_{0}}{\partial S_{\mathrm{cp}}}=\frac{\Delta_{0}}{2 p_{0}}\left(2 \frac{\partial p_{0}}{\partial S_{\mathrm{cp}}}+\operatorname{Tr}\left(\mathbf{A}_{\perp}^{(\mathrm{L})}-\mathbf{A}_{\perp}^{(\mathrm{R})}\right)-\operatorname{Tr}\left(\mathbf{A}^{(\mathrm{L})}-\mathbf{A}^{(\mathrm{R})}\right)\right) .
$$

Finally, since $\operatorname{Tr} \mathbf{A}^{(\mathrm{L})}=p_{0}^{\prime}+\operatorname{Tr} \mathbf{A}_{\perp}^{(\mathrm{L})}$ and $\operatorname{Tr} \mathbf{A}^{(\mathrm{R})}=-p_{0}^{\prime}+\operatorname{Tr} \mathbf{A}_{\perp}^{(\mathrm{R})}$, as shown in Ref. 52, we have

$$
\begin{aligned}
\frac{\partial \Delta_{0}}{\partial S_{\mathrm{cp}}}= & \frac{\Delta_{0}}{2 p_{0}}\left(2 \frac{\partial p_{0}}{\partial S_{\mathrm{cp}}}+\operatorname{Tr}\left(\mathbf{A}_{\perp}^{(\mathrm{L})}-\mathbf{A}_{\perp}^{(\mathrm{R})}\right)\right. \\
& \left.-\operatorname{Tr}\left(\mathbf{A}_{\perp}^{(\mathrm{L})}-\mathbf{A}_{\perp}^{(\mathrm{R})}\right)-2 \frac{\partial p_{0}}{\partial S_{\mathrm{cp}}}\right)=0,
\end{aligned}
$$

which proves that the ground-state tunneling splitting does not depend on $S_{\mathrm{cp}}$, the position of the dividing plane.
Similarly, the invariance of the excited-state tunneling splitting on the position of the dividing plane is checked by differentiating Eq. (33) with respect to $S_{\mathrm{cp}}$. If only the $F$ terms are included in the expansion of $\exp (-w)$, we have

$$
\frac{\partial \Delta_{1}}{\partial S_{\mathrm{cp}}}=\Delta_{0} 2 \omega_{\mathrm{e}}\left(\frac{\partial F^{(\mathrm{L})}}{\partial S_{\mathrm{cp}}} F^{(\mathrm{R})}+F^{(\mathrm{L})} \frac{\partial F^{(\mathrm{R})}}{\partial S_{\mathrm{cp}}}\right),
$$

which together with Eq. (21) gives

$$
\frac{\partial \Delta_{1}}{\partial S_{\mathrm{cp}}}=\Delta_{0} 2 \omega_{\mathrm{e}}\left(\frac{\omega_{\mathrm{e}}}{p_{0}} F^{(\mathrm{L})} F^{(\mathrm{R})}-\frac{\omega_{\mathrm{e}}}{p_{0}} F^{(\mathrm{L})} F^{(\mathrm{R})}\right)=0 .
$$

If we include the $\mathbf{U}$ terms in the expansion of $\exp (-w)$, the derivative of the splitting becomes

$$
\begin{aligned}
\frac{\partial \Delta_{1}}{\partial S_{\mathrm{cp}}}= & \Delta_{0} \omega_{\mathrm{e}}\left(\frac{\partial}{\partial S_{\mathrm{cp}}} \mathbf{U}^{(\mathrm{L}) \top} \overline{\mathbf{A}}^{-1} \mathbf{U}^{(\mathrm{R})}+\mathbf{U}^{(\mathrm{L}) \top} \frac{\partial}{\partial S_{\mathrm{cp}}} \overline{\mathbf{A}}^{-1} \mathbf{U}^{(\mathrm{R})}\right. \\
& \left.+\mathbf{U}^{(\mathrm{L}) \top} \overline{\mathbf{A}}^{-1} \frac{\partial}{\partial S_{\mathrm{cp}}} \mathbf{U}^{(\mathrm{R})}\right) .
\end{aligned}
$$

It can be shown that

$$
p_{0} \frac{\partial}{\partial S_{\mathrm{cp}}} \overline{\mathbf{A}}^{-1}=\mathbf{P} \mathbf{A}^{(\mathrm{L})} \overline{\mathbf{A}}^{-1}-\overline{\mathbf{A}}^{-1} \mathbf{A}^{(\mathrm{R})} \mathbf{P},
$$

which can be used to rewrite Eq. (D9) as

$$
\begin{aligned}
\frac{\partial \Delta_{1}}{\partial S_{\mathrm{cp}}}= & \Delta_{0} \frac{\omega_{\mathrm{e}}}{p_{0}}\left(-\left(\mathbf{U}^{(\mathrm{L})}-\mathbf{P} \mathbf{U}^{(\mathrm{L})}\right)^{\top} \mathbf{A}^{(\mathrm{L})} \overline{\mathbf{A}}^{-1} \mathbf{U}^{(\mathrm{R})}\right. \\
& \left.+\mathbf{U}^{(\mathrm{L}) \top} \overline{\mathbf{A}}^{-1} \mathbf{A}^{(\mathrm{R})}\left(\mathbf{U}^{(\mathrm{R})}-\mathbf{P} \mathbf{U}^{(\mathrm{R})}\right)\right) .
\end{aligned}
$$

In this form, it is evident that if $\mathbf{U}$ remains orthogonal to the instanton path, i.e., $\mathbf{P U}=\mathbf{U}$, the excited-state tunneling splittings become independent of the position of the dividing plane. If that is not the case, however, Eq. (D9) can be further simplified to

$$
\begin{aligned}
\frac{\partial \Delta_{1}}{\partial S_{\mathrm{cp}}}= & \Delta_{0} \frac{\omega_{\mathrm{e}}}{p_{0}}\left(-\frac{\omega_{\mathrm{e}}}{p_{0}} F^{(\mathrm{L})} \mathbf{t}^{\top} \mathbf{A}^{(\mathrm{L})} \overline{\mathbf{A}}^{-1} \mathbf{U}^{(\mathrm{R})}\right. \\
& \left.-\frac{\omega_{\mathrm{e}}}{p_{0}} F^{(\mathrm{R})} \mathbf{U}^{(\mathrm{L}) \top} \overline{\mathbf{A}}^{-1} \mathbf{A}^{(\mathrm{R})} \mathbf{t}\right)
\end{aligned}
$$

which does not vanish and the tunneling splitting will, in general, depend on the position of the dividing plane, as observed in Sec. IV.

If the same analysis is performed with the $\mathbf{Z}$ terms, there arise two factors that cancel out the $\mathbf{U}$ terms. However, a multitude of other factors also arise, which again cause the dependence on the position of the dividing plane. As mentioned above, the root of the problem is that the expansion of $\exp (-w)$ is inconsistent with the expansion of $W_{1}$, and it gives rise to terms of all orders in $\Delta \mathbf{x}$. However, in the case of a symmetric potential, all perpendicular components of the gradients, Hessians, and third-order tensors are the same for the left- and right-localized wavefunctions at the dividing plane in the middle of the instanton path, while their tangent components differ in sign. Thus, it is possible to show that the derivative of the $\mathbf{Z}$ contribution with respect to $S_{\mathrm{cp}}$ vanishes at the middle of the instanton path, and numerical tests show that its contribution is minimal there. Therefore, for symmetric systems, the middle of 
the path represents the optimal position of the dividing plane. For the asymmetric paths, there is no such preferential point on the instanton. However, good results are obtained by positioning the dividing plane at the maximum of the barrier as, at this point, $p_{0}$ is the largest and the derivatives of the splitting are generally smallest, which means that, at this point, the splittings are relatively stable.

\section{DATA AVAILABILITY}

The data that support the findings of this study are available from the corresponding author upon reasonable request.

\section{REFERENCES}

${ }^{1}$ R. P. Bell, The Tunnel Effect in Chemistry (Chapman and Hall, London, 1980).

${ }^{2}$ L. H. Coudert and J. T. Hougen, J. Mol. Spectrosc. 130, 86 (1988).

${ }^{3}$ T. R. Walsh and D. J. Wales, J. Chem. Soc. Faraday Trans. 92, 2505 (1996).

${ }^{4}$ Y. Xu and W. Jäger, J. Chem. Phys. 106, 7968 (1997).

${ }^{5}$ F. N. Keutsch and R. J. Saykally, Proc. Natl. Acad. Sci. U. S. A. 98, 10533 (2001).

${ }^{6}$ K. Liu, M. G. Brown, J. D. Cruzan, and R. J. Saykally, Science 271, 62 (1996).

${ }^{7}$ M. T. Cvitaš and J. O. Richardson, in Molecular Spectroscopy and Quantum Dynamics, edited by R. Marquardt and M. Quack (Elsevier, 2020), Chap. 10.

${ }^{8}$ T. Hammer, M. D. Coutinho-Neto, A. Viel, and U. Manthe, J. Chem. Phys. 131, 224109 (2009)

${ }^{9}$ C. Fábri, R. Marquardt, A. G. Császár, and M. Quack, J. Chem. Phys. 150, 014102 (2019).

${ }^{10}$ P. M. Felker and Z. Bačić, J. Chem. Phys. 151, 024305 (2019).

${ }^{11}$ M. Ceriotti, W. Fang, P. G. Kusalik, R. H. McKenzie, A. Michaelides, M. A. Morales, and T. E. Markland, Chem. Rev. 116, 7529 (2016).

${ }^{12}$ Y. Wang, X. Huang, B. C. Shepler, B. J. Braams, and J. M. Bowman, J. Chem, Phys. 134, 094509 (2011).

${ }^{13}$ S. K. Reddy, S. C. Straight, P. Bajaj, C. Huy Pham, M. Riera, D. R. Moberg, M. A. Morales, C. Knight, A. W. Götz, and F. Paesani, J. Chem. Phys. 145, 194504 (2016).

${ }^{14}$ J. O. Richardson, S. C. Althorpe, and D. J. Wales, J. Chem. Phys. 135, 124109 (2011).

${ }^{15}$ F. N. Keutsch, R. S. Fellers, M. R. Viant, and R. J. Saykally, J. Chem. Phys. 114, 4005 (2001).

${ }^{16}$ W. T. S. Cole, R. S. Fellers, M. R. Viant, and R. J. Saykally, J. Chem. Phys. 146, 014306 (2017)

${ }^{17}$ M. T. Cvitaš and J. O. Richardson, Phys. Chem. Chem. Phys. 22, 1035 (2019).

${ }^{18}$ B. J. Mhin, J. Kim, S. Lee, J. Y. Lee, and K. S. Kim, J. Chem. Phys. 100, 4484 (1994).

${ }^{19}$ C. Pérez, M. T. Muckle, D. P. Zaleski, N. A. Seifert, B. Temelso, G. C. Shields, Z. Kisiel, and B. H. Pate, Science 336, 897 (2012).

${ }^{20}$ J. O. Richardson, C. Pérez, S. Lobsiger, A. A. Reid, B. Temelso, G. C. Shields, Z. Kisiel, D. J. Wales, B. H. Pate, and S. C. Althorpe, Science 351, 1310 (2016).

${ }^{21}$ C. Léonard, N. C. Handy, S. Carter, and J. M. Bowman, Spectrochim. Acta, Part A 58, 825 (2002).

${ }^{22}$ M. Neff and G. Rauhut, Spectrochim. Acta, Part A 119, 100 (2014).

${ }^{23}$ J. Šmydke, C. Fábri, J. Sarka, and A. G. Császár, Phys. Chem. Chem. Phys. 21, 3453 (2019).

${ }^{24}$ F. Wu, Y. Ren, and W. Bian, J. Chem. Phys. 145, 074309 (2016).

${ }^{25}$ C. Leforestier, K. Szalewicz, and A. van der Avoird, J. Chem. Phys. 137, 014305 (2012).

${ }^{26}$ X.-G. Wang and T. Carrington, J. Chem. Phys. 148, 074108 (2018).

${ }^{27}$ M. Schröder, F. Gatti, and H.-D. Meyer, J. Chem. Phys. 134, 234307 (2011).

${ }^{28}$ M. Schröder and H.-D. Meyer, J. Chem. Phys. 141, 034116 (2014).

${ }^{29}$ T. Hammer and U. Manthe, J. Chem. Phys. 134, 224305 (2011).

${ }^{30}$ D. Blume and K. B. Whaley, J. Chem. Phys. 112, 2218 (2000).

${ }^{31}$ A. Viel, M. D. Coutinho-Neto, and U. Manthe, J. Chem. Phys. 126, 024308 (2007).
${ }^{32}$ Y. Wang, B. J. Braams, J. M. Bowman, S. Carter, and D. P. Tew, J. Chem. Phys. 128, 224314 (2008).

${ }^{33}$ C. L. Vaillant, D. J. Wales, and S. C. Althorpe, J. Phys. Chem. Lett. 10, 7300 (2019).

${ }^{34}$ D. J. Nesbitt and F. Dong, Phys. Chem. Chem. Phys. 10, 2113 (2008).

${ }^{35} \mathrm{C} . \mathrm{Qu}$ and J. M. Bowman, Phys. Chem. Chem. Phys. 18, 24835 (2016).

${ }^{36}$ S. C. Althorpe and D. C. Clary, J. Chem. Phys. 102, 4390 (1995).

${ }^{37}$ I. Matanović, N. Došlić, and B. R. Johnson, J. Chem. Phys. 128, 084103 (2008).

${ }^{38}$ E. Kamarchik, Y. Wang, and J. Bowman, J. Phys. Chem. A 113, 7556 (2009).

${ }^{39}$ T. D. Sewell, Y. Guo, and D. L. Thompson, J. Chem. Phys. 103, 8557 (1995).

${ }^{40}$ C. S. Tautermann, A. F. Voegele, T. Loerting, and K. R. Liedl, J. Chem. Phys. 117, 1967 (2002).

${ }^{41}$ M. Ceotto, Mol. Phys. 110, 547 (2012).

${ }^{42}$ T. A. H. Burd and D. C. Clary, J. Chem. Theory Comput. 16, 3486 (2020).

${ }^{43}$ N. Makri and W. H. Miller, J. Chem. Phys. 91, 4026 (1989).

${ }^{44}$ S. Coleman, in Proceedings International School of Subnuclear Physics (Erice, 1977); Also in Aspects of Symmetry (Cambridge University Press, 1985), Chap. 7 , pp. 265-350.

${ }^{45}$ A. I. Vainshtein, V. I. Zakharov, V. A. Novikov, and M. A. Shifman, Sov. Phys, Usp. 25, 195 (1982); Also in Instantons in Gauge Theories, edited by M. Shifman (World Scientific, Singapore, 1994), p. 468.

${ }^{46}$ W. H. Miller, J. Chem. Phys. 62, 1899 (1975).

${ }^{47}$ V. A. Benderskii, D. E. Makarov, and C. A. Wight, Chemical Dynamics at Low Temperatures, Advances in Chemical Physics Vol. 88 (Wiley, New York, 1994).

${ }^{48}$ W. Siebrand, Z. Smedarchina, M. Z. Zgierski, and A. Fernández-Ramos, Int. Rev. Phys. Chem. 18, 5 (1999).

${ }^{49}$ Z. Smedarchina, W. Caminati, and F. Zerbetto, Chem. Phys. Lett. 237, 279 (1995).

${ }^{50} \mathrm{Z}$. Smedarchina, W. Siebrand, and A. Fernández-Ramos, J. Chem. Phys. 137, 224105 (2012).

${ }^{51}$ V. A. Benderskii, E. V. Vetoshkin, S. Yu. Grebenshchikov, L. von Laue, and H. P. Trommsdorff, Chem. Phys. 219, 119 (1997).

${ }^{52}$ G. V. Mil'nikov and H. Nakamura, J. Chem. Phys. 115, 6881 (2001).

${ }^{53}$ G. V. Mil'nikov and H. Nakamura, J. Chem. Phys. 122, 124311 (2005).

${ }^{54}$ J. O. Richardson and S. C. Althorpe, J. Chem. Phys. 134, 054109 (2011).

${ }^{55}$ C. L. Vaillant and M. T. Cvitaš, Phys. Chem. Chem. Phys. 20, 26809 (2018).

${ }^{56}$ J. O. Richardson, D. J. Wales, S. C. Althorpe, R. P. McLaughlin, M. R. Viant, O. Shih, and R. J. Saykally, J. Phys. Chem. A 117, 6960 (2013).

${ }^{57}$ A. Garg, Am. J. Phys. 68, 430 (2000).

${ }^{58}$ C. Herring, Rev. Mod. Phys. 34, 631 (1962).

${ }^{59}$ L. D. Landau and E. M. Lifshitz, Quantum Mechanics: Non-Relativistic Theory, 2nd ed. (Pergamon Press, Oxford, 1965).

${ }^{60} \mathrm{H}$. Nakamura and G. Mil'nikov, Quantum Mechanical Tunneling in Chemical Physics (CRC Press, Boca Raton, FL, 2013).

${ }^{61}$ V. A. Benderskii, E. V. Vetoshkin, L. von Laue, and H. P. Trommsdorff, Chem. Phys. 219, 143 (1997).

${ }^{62}$ V. A. Benderskii, E. V. Vetoshkin, I. S. Irgibaeva, and H. P. Trommsdorff, Chem. Phys. 262, 393 (2000).

${ }^{63}$ Z. Smedarchina, W. Siebrand, and M. Z. Zgierski, J. Chem. Phys. 104, 1203 (1996).

${ }^{64}$ A. Fernández-Ramos, Z. Smedarchina, M. Z. Zgierski, and W. Siebrand, J. Chem. Phys. 109, 1004 (1998).

${ }^{65}$ G. Mil'nikov, O. Kühn, and H. Nakamura, J. Chem. Phys. 123, 074308 (2005).

${ }^{66}$ G. V. Mil'nikov, T. Ishida, and H. Nakamura, J. Phys. Chem. A 110, 5430 (2006).

${ }^{67}$ M. Eraković, C. L. Vaillant, and M. T. Cvitaš, J. Chem. Phys. 152, 084111 (2020).

${ }^{68} \mathrm{M}$. T. Cvitaš and S. C. Althorpe, J. Chem. Theory Comput. 12, 787 (2016).

${ }^{69}$ M. T. Cvitaš, J. Chem. Theory Comput. 14, 1487 (2018).

${ }^{70} \mathrm{H}$. Kleinert, Path Integrals in Quantum Mechanics, Statistics, Polymer Physics and Financial Markets, 5th ed. (World Scientific, Singapore, 2009).

${ }^{71}$ V. A. Benderskii, S. Y. Grebenshchikov, and G. V. Mil'nikov, Chem. Phys. 194, 1 (1995). 
${ }^{72}$ W. H. Press, S. A. Teukolsky, W. T. Vetterling, and B. P. Flannery, Numerical Recipes: The Art of Scientific Computing, 3rd ed. (Cambridge University Press, Cambridge, 2007).

${ }^{73}$ T. Kawatsu and S. Miura, J. Chem. Phys. 141, 024101 (2014).

${ }^{74}$ J. C. Light, I. P. Hamilton, and J. V. Lill, J. Chem. Phys. 82, 1400 (1985).

${ }^{75}$ V. Babin, C. Leforestier, and F. Paesani, J. Chem. Theory Comput. 9, 5395 (2013).

${ }^{76}$ V. Babin, G. R. Medders, and F. Paesani, J. Chem. Theory Comput. 10, 1599 (2014).

${ }^{77}$ C. L. Vaillant, S. C. Althorpe, and D. J. Wales, J. Chem. Theory Comput. 15, 33 (2019).
${ }^{78}$ Y. Watanabe, T. Taketsugu, and D. J. Wales, J. Chem. Phys. 120, 5993 (2004).

${ }^{79}$ L. B. Braly, J. D. Cruzan, K. Liu, R. S. Fellers, and R. J. Saykally, J. Chem. Phys. 112, 10293 (2000).

${ }^{80}$ E. N. Karyakin, G. T. Fraser, and R. D. Suenram, Mol. Phys. 78, 1179 (1993).

${ }^{81}$ T. T. Nguyen, E. Székely, G. Imbalzano, J. Behler, G. Csányi, M. Ceriotti, A. W. Götz, and F. Paesani, J. Chem. Phys. 148, 241725 (2018).

${ }^{82} \mathrm{R}$. Courant and D. Hilbert, Methods of Mathematical Physics (WileyInterscience, 1962), Vol. II.

${ }^{83}$ R. M. Minyaev and D. J. Wales, J. Chem. Soc., Faraday Trans. 90, 1839 (1994). 